\title{
Analysis of ionospheric electrodynamic parameters on mesoscales - a review of selected techniques using data from ground-based observation networks and satellites
}

\author{
H. Vanhamäki ${ }^{1, *}$ and O. Amm ${ }^{1}$ \\ ${ }^{1}$ Arctic Research Unit, Finnish Meteorological Institute, Helsinki, Finland \\ *visiting at: Solar-Terrestrial Environment Laboratory, Nagoya University, Nagoya, Japan \\ Received: 9 April 2010 - Revised: 2 November 2010 - Accepted: 28 February 2011 - Published: 4 March 2011
}

\begin{abstract}
We present a review of selected data-analysis methods that are frequently applied in studies of ionospheric electrodynamics and magnetosphere-ionosphere coupling using ground-based and space-based data sets. Our focus is on methods that are data driven (not simulations or statistical models) and can be used in mesoscale studies, where the analysis area is typically some hundreds or thousands of $\mathrm{km}$ across. The selection of reviewed methods is such that most combinations of measured input data (electric field, conductances, magnetic field and currents) that occur in practical applications are covered. The techniques are used to solve the unmeasured parameters from Ohm's law and Maxwell's equations, possibly with help of some simplifying assumptions. In addition to reviewing existing data-analysis methods, we also briefly discuss possible extensions that may be used for upcoming data sets.
\end{abstract}

Keywords. Ionosphere (Auroral ionosphere; Electric fields and currents; Ionosphere-magnetosphere interactions)

\section{Introduction}

We present a review of selected data-analysis methods that are applied in studies of ionospheric electrodynamics and magnetosphere-ionosphere coupling using ground-based and space-based data sets. At present, there is no single measurement device that can measure all ionospheric electrodynamic parameters directly and simultaneously, with good spatial and temporal resolution and coverage. Therefore data-analysis techniques are needed to combine different

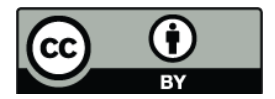

Correspondence to: $\mathrm{H}$. Vanhamäki (heikki.vanhamaki@fmi.fi) types of measured data and to obtain unobserved ionospheric parameters from the observed ones, possibly using some additional assumptions in the process. We concentrate on methods that are data driven and applicable to single events (not simulations or statistical models), and which can be used in mesoscale studies, where the analysis area is typically some hundreds or thousands of $\mathrm{km}$ across.

The primary focus of this review is in ionospheric electrodynamics, so we do not include variables like chemical composition, temperature, etc. in our discussion. Furthermore we concentrate on analysis techniques that have been developed to be used with data from the MIRACLE network (Magnetometers - Ionospheric Radars - All-sky Cameras Large Experiment, Syrjäsuo et al., 1998) illustrated in Fig. 1, possibly in combination with satellite observations, for example Cluster (Escoubet et al., 2001) or CHAMP (Ritter et al., 2004). However, the techniques can be applied to data from any other mesoscale network with similar observations.

Table 1 gives an overview of the data-analysis methods that are reviewed here. For each method, we list the input data, additional assumptions (if any) required for the method to be applicable and the output produced. The full set of ionospheric electrodynamic parameters that can be calculated consist of the ionospheric horizontal electric field $\boldsymbol{E}$, height integrated Hall and Pedersen conductances $\Sigma_{\mathrm{H}}$ and $\Sigma_{\mathrm{P}}$, horizontal current $\boldsymbol{J}$ and field aligned current (FAC) $j_{\|}$. Additionally, the ground magnetic perturbation field $\boldsymbol{B}_{\mathrm{G}}$ is an important input parameter in many analysis methods.

Most of the methods listed in Table 1 are used in 2dimensional (latitude-longitude) regions of the ionosphere, but some methods have also 1-dimensional variants, as indicated by $*$ in the last column of the table. In 1-D analysis it is assumed that ionospheric parameters vary only in one horizontal direction (e.g. as a function of geomagnetic latitude),

Published by Copernicus Publications on behalf of the European Geosciences Union. 


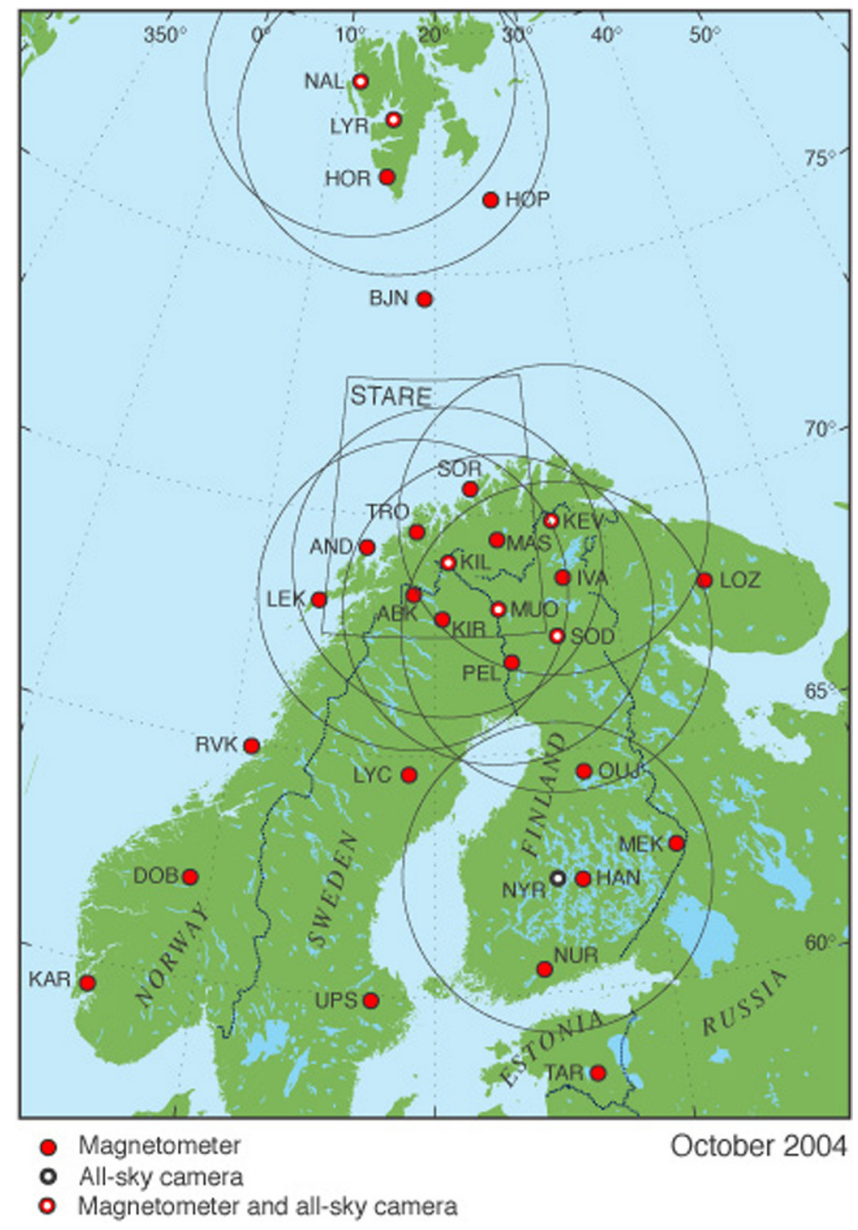

Fig. 1. The MIRACLE instrument network. Circles give the field of view of each all-sky camera. Also the combined field of view of the STARE radars (decommissioned in May 2005) is shown.

so input data is required along a single chain or a satellite track only. The 1-D methods are especially useful when analyzing data from an overpassing satellite or from a meridional magnetometer chain.

Some of the methods listed Table 1 have been discussed by Glassmeier (1987) and Untiedt and Baumjohann (1993), who also give application examples and references to older studies where the methods have been utilized. In the present review we will concentrate on more recent work done during the last two decades. Also, some classical techniques, such as magnetic field separation and upward continuation (e.g. Chapman and Bartels, 1940; Haines, 1985), have seen significant improvement during this period.

In Sect. 2 we review basic electrodynamic properties of ionospheric current systems, as well as the most commonly used approximations. In the following sections we discuss the selected analysis methods listed in Table 1, grouped according to the primary output they produce. Methods to determine equivalent currents $\boldsymbol{J}_{\text {eq }}$ and total current $\boldsymbol{J}$ are re- viewed in Sects. 3 and 4, respectively. Sections 5 and 6 discuss methods to estimate the electric field $\boldsymbol{E}$ or conductances $\Sigma_{\mathrm{H}}$ and $\Sigma_{\mathrm{P}}$. The 1-dimensional variants of some methods are discussed separately in Sect. 7 and ways to include effects of electromagnetic induction in data-analysis tools are reviewed in Sect. 8. In a final outlook we briefly discuss some extension of presently used analysis methods and future possibilities offered by new instrumentation, such as the Swarm satellite mission and EISCAT 3-D radar.

\section{Mathematical background}

In this section we review the basic properties of ionospheric electrodynamics, especially at high magnetic latitudes (i.e., the auroral oval). We employ the commonly used thinsheet approximation (see e.g. Glassmeier, 1987; Untiedt and Baumjohann, 1993), where the ionosphere is assumed to be a thin, 2-dimensional spherical shell of radius $R_{\mathrm{I}}$ at a constant altitude above the Earth. This approximation is justified by the fact that the largest horizontal currents flow at about $100-125 \mathrm{~km}$ altitude, in a layer that is much thinner than the horizontal extents typically considered. However, in some cases 3-dimensional modeling is required (Amm et al., 2008). Another occasionally used approximation is to neglect the curvature of the ionosphere on areas less than $\sim 1000 \mathrm{~km}$ across and to use Cartesian instead of spherical geometry (e.g. Sect. 2.1 of Untiedt and Baumjohann, 1993).

The main electrodynamic variables are: horizontal sheet current density $\boldsymbol{J}$, field-aligned current $j_{\|}$, horizontal electric field $\boldsymbol{E}$, magnetic field $\boldsymbol{B}$ and height integrated Hall and Pedersen conductances $\Sigma_{\mathrm{H}}$ and $\Sigma_{\mathrm{P}}$. These variables are related through Maxwell's equations, Ohm's law and current continuity,

$(\nabla \times \boldsymbol{E})_{\mathrm{r}}=-\frac{\partial B_{\mathrm{r}}}{\partial t}$

$\nabla \times \boldsymbol{B}=\mu_{0} \boldsymbol{j}=\mu_{0} \boldsymbol{J} \delta\left(r-R_{\mathrm{I}}\right)-\mu_{0} j_{\|} \hat{\boldsymbol{e}}_{\mathrm{r}}$

$\boldsymbol{J}=\Sigma_{\mathrm{P}} \boldsymbol{E}-\Sigma_{\mathrm{H}} \hat{\boldsymbol{e}}_{\mathrm{r}} \times \boldsymbol{E}$

$j_{\|}=\nabla \cdot J$.

In Eqs. (1)-(4) we have made the frequently used assumption of a radial magnetic field, so that $\hat{\boldsymbol{e}}_{\|}=-\hat{\boldsymbol{e}}_{\mathrm{r}}$ at the Northern Hemisphere. According to Untiedt and Baumjohann (1993) and Amm (1998) the effect of the tilted field lines is negligible for inclination angles $\chi \gtrsim 75^{\circ}$, which covers the auroral zone. Elsewhere the inclination of the magnetic field can be taken into account by modifying the Hall and Pedersen conductances in Eq. (3) (see e.g. Brekke, 1997, chapter 7.12) and by calculating the FAC as $j_{\|}=\nabla \cdot \boldsymbol{J} / \sin \chi$.

\subsection{Equations and unknowns}

We may now count the number of electrodynamic parameters we are interested in and the number of equations connecting 
Table 1. Overview of selected analysis methods.

\begin{tabular}{ccccc}
\hline Input & Assumptions & Output & Name of method & 1-D \\
\hline $\boldsymbol{B}_{\mathrm{G}}$ & - & $\boldsymbol{J}_{\text {eq,ion }}, \boldsymbol{J}_{\text {eq, int }}$ & $\begin{array}{c}\text { Field continuation } \\
\text { and separation }\end{array}$ & $*$ \\
\hline $\boldsymbol{B}_{\mathrm{G}}, j_{\|},\{\boldsymbol{E}\}$ & - & $\boldsymbol{J},\left\{\Sigma_{\mathrm{P}}, \Sigma_{\mathrm{H}}\right\}$ & $\begin{array}{c}\text { Elementary current } \\
\text { method }\end{array}$ & $*$ \\
\hline $\boldsymbol{B}_{G}, \Sigma_{\mathrm{P}}, \Sigma_{\mathrm{H}}$ & $\begin{array}{c}\text { Usually } \\
\nabla \times \boldsymbol{E}=0\end{array}$ & $\boldsymbol{E}, \boldsymbol{J}, j_{\|}$ & KRM & \\
\hline$j_{\|}, \Sigma_{\mathrm{P}}, \Sigma_{\mathrm{H}}$ & $\begin{array}{c}\text { Usually } \\
\nabla \times \boldsymbol{E}=0\end{array}$ & $\boldsymbol{E}, \boldsymbol{J}$ & - & \\
\hline $\boldsymbol{B}_{\mathrm{G}}, \boldsymbol{E}$ & $\begin{array}{c}\alpha=\Sigma_{\mathrm{H}} / \Sigma_{\mathrm{P}} \\
j_{\|}, \boldsymbol{E}\end{array}$ & $\Sigma_{\mathrm{H}}, \Sigma_{\mathrm{P}}, \boldsymbol{J}, j_{\|}$ & $\begin{array}{c}\text { Method of } \\
\text { characteristics } \\
\left(\boldsymbol{J}_{\text {eq }} \text {-based) }\right. \\
\text { (FAC-based })\end{array}$ & $*$ \\
\hline $\boldsymbol{B}_{\text {satellite }}$ & 1-dimensional & $\boldsymbol{J}, j_{\|}$ & 1-D SECS & $*$ \\
\hline
\end{tabular}

them. In a thin sheet ionosphere the electric field $\boldsymbol{E}$ and horizontal current $\boldsymbol{J}$ are 2-dimensional vector fields that can be represented by two potentials,

$\boldsymbol{E}=-\nabla \phi_{E}-\hat{\boldsymbol{e}}_{\mathrm{r}} \times \nabla \psi_{E}$

$\boldsymbol{J}=-\nabla \phi_{J}-\hat{\boldsymbol{e}}_{\mathrm{r}} \times \nabla \psi_{J}$

The function $\phi_{E}$ is the usual electrostatic potential and $\psi_{E}$ is related to the rotational inductive part of the electric field. It is usually assumed that $\nabla \psi_{E}=0$, but this does not hold in some situations, as discussed in Sect. 8. The current potential $\phi_{J}$ is connected to FAC through Eq. (4) while $\psi_{J}$ is related to equivalent current and ground magnetic disturbance, as discussed in Sects. 2.2 and 3 below.

The following six 2-dimensional scalar fields specify the electrodynamic state of a thin sheet ionosphere:

$\phi_{E}, \psi_{E}, \phi_{J}, \psi_{J}, \Sigma_{\mathrm{H}}, \Sigma_{\mathrm{H}}$.

The curl and divergence of Ohm's law give us two scalar equations that relate the variables (Untiedt, 1983; Glassmeier, 1987; Untiedt and Baumjohann, 1993),

$$
\begin{aligned}
(\nabla \times \boldsymbol{J})_{\mathrm{r}}= & \left(\nabla \Sigma_{\mathrm{P}} \times \boldsymbol{E}\right)_{\mathrm{r}}+\Sigma_{\mathrm{P}}(\nabla \times \boldsymbol{E})_{\mathrm{r}}-\nabla \Sigma_{\mathrm{H}} \cdot \boldsymbol{E}- \\
& -\Sigma_{\mathrm{H}} \nabla \cdot \boldsymbol{E}, \\
\nabla \cdot \boldsymbol{J}= & \nabla \Sigma_{\mathrm{P}} \cdot \boldsymbol{E}+\Sigma_{\mathrm{P}} \nabla \cdot \boldsymbol{E}+\left(\nabla \Sigma_{\mathrm{H}} \times \boldsymbol{E}\right)_{\mathrm{r}}+ \\
& +\Sigma_{\mathrm{H}}(\nabla \times \boldsymbol{E})_{\mathrm{r}} .
\end{aligned}
$$

A third equation is obtained by combining Ampere's and Faraday's laws, which relate the curl of electric field to the electric current. It follows that we have to know at least 3 of the 6 variables listed in Eq. (7) in order to solve for the ionospheric electrodynamic state. This is reflected also in Table 1, where e.g. the method of characteristics requires 3 known input parameters.

\subsection{The concept of equivalent current}

The ground magnetic data $\boldsymbol{B}_{\mathrm{G}}$ is often most conveniently used in the form of an equivalent current. By definition, ionospheric equivalent current $\boldsymbol{J}_{\text {eq,ion }}$ is a 2-dimensional, divergence-free sheet current that produces the same ground magnetic field as the real 3-dimensional system consisting of ionospheric currents and FAC. According to potential theory, this kind of equivalent current solution always exists and is uniquely defined in global scale (see discussion in Haines and Torta, 1994). Using field continuation and, if necessary, field separation techniques (see Sect. 3), the ionospheric equivalent current $\boldsymbol{J}_{\text {eq,ion }}$ can be derived from measured $\boldsymbol{B}_{\mathrm{G}}$.

In addition to external ionospheric and magnetospheric currents, there are also internal sources of magnetic variations. Changes in external currents create an induced electric field, according to Faraday's law. The induced field drives currents in the ground, depending on the conductivity of the local bedrock. This process of geomagnetic induction distorts the original magnetic signal from external sources, and makes analysis more difficult (e.g. Untiedt and Baumjohann, 1993). The magnetic variations that are caused by the internal induced sources can be separately represented using internal equivalent currents $\boldsymbol{J}_{\text {eq,int }}$, analogous to $\boldsymbol{J}_{\text {eq,ion }}$.

An important question about the ionospheric equivalent currents is their relation to the real currents. The true sheet current density $\boldsymbol{J}$ can be divided into 3 parts, curl-free (potential), divergence-free (rotational) and Laplacian, so that

$\boldsymbol{J}=\boldsymbol{J}_{\text {pot }}+\boldsymbol{J}_{\text {rot }}+\boldsymbol{J}_{\text {Laplace }}$,

where

$$
\begin{gathered}
\nabla \cdot J_{\text {rot }}=0, \quad \nabla \cdot J_{\text {Laplace }}=0, \\
\left(\nabla \times J_{\text {pot }}\right)_{\mathrm{r}}=0, \quad\left(\nabla \times J_{\text {Laplace }}\right)_{\mathrm{r}}=0 .
\end{gathered}
$$


The Laplacian part $\boldsymbol{J}_{\text {Laplace }}$ represents that part of the current that has no divergence or curl inside the analysis area. In global analysis $\boldsymbol{J}_{\text {Laplace }}=0$, and in mesoscale studies it typically is a homogeneous background current (see Sect. 2.4.2 of Untiedt and Baumjohann, 1993).

If the background magnetic field is perpendicular to the ionospheric plane, then the true curl-free current system $\boldsymbol{J}_{\text {pot }}$ together with associated FAC does not produce any magnetic field below the ionosphere. Fukushima (1976) derived this result by assuming uniform ionospheric conductances, but the result is valid independently of the conductance distribution (Amm, 1997). As $\boldsymbol{J}_{\text {eq,ion }}$ can be uniquely defined as the divergence-free sheet current that produce the observed magnetic field below the ionosphere, it follows that equivalent currents are equal to the divergence-free part of the true currents,

$J_{\text {rot }}=J_{\text {eq,ion }}$.

This approximation is only valid for inclination angles $\chi \gtrsim$ $75^{\circ}$. This is a common limitation for all the analysis methods listed in Table 1 that use $\boldsymbol{B}_{\mathrm{G}}$ as input data.

Usually Eq. (11) is all we can say about the real ionospheric currents using just ground magnetic data. In some special cases we may get a rough estimate of the FAC directly from the equivalent currents, by assuming that conductivity gradients are parallel to the electric field and the ratio $\alpha=\Sigma_{\mathrm{H}} / \Sigma_{\mathrm{P}}$ is a constant. This line of reasoning was applied by Amm et al. (2002) for a pair of traveling convection vortices, which exhibited a high degree of symmetry. However, the required assumptions are usually too restrictive to apply.

\subsection{Elementary current systems}

Elementary current systems were introduced by Amm (1997). Although for historical reasons their name refers to currents, they can be used to represent any 2-dimensional vector field. Elementary current systems can be defined either in spherical or Cartesian geometry, and they are called SECS and CECS, respectively. There are two different types of elementary systems, one is divergence-free (DF) and the other curl-free (CF). The spherical CF and DF elementary systems, shown in Fig. 2, are defined as

$\mathbf{J}_{\mathrm{CF}}\left(\boldsymbol{r}^{\prime}\right)=\frac{I_{\mathrm{CF}}}{4 \pi R} \cot \left(\frac{\theta^{\prime}}{2}\right) \hat{\boldsymbol{e}}_{\theta^{\prime}}$

$\mathbf{J}_{\mathrm{DF}}\left(\boldsymbol{r}^{\prime}\right)=\frac{I_{\mathrm{DF}}}{4 \pi R} \cot \left(\frac{\theta^{\prime}}{2}\right) \hat{\boldsymbol{e}}_{\phi^{\prime}}$

Here $I_{\mathrm{DF}}$ and $I_{\mathrm{DF}}$ are the scaling factors of the elementary systems, while $R$ is the radius of the sphere (e.g. ionosphere) where elementary systems are placed. The expressions are given in a spherical coordinate system $\left(r^{\prime}, \theta^{\prime}, \phi^{\prime}\right)$, with unit vectors $\left(\hat{\boldsymbol{e}}_{r^{\prime}}, \hat{\boldsymbol{e}}_{\theta^{\prime}}, \hat{\boldsymbol{e}}_{\phi^{\prime}}\right)$, that has its pole at the center of the elementary systems. The CF and DF elementary systems form a complete set of basis functions for representing 2-dimensional vector fields on a sphere (SECS) or on a plane (CECS). A short guide, together with further references about the use of elementary systems, is given in appendix A of Vanhamäki (2007).

The elementary systems are defined in such a way that the CF system has a Dirac $\delta$-function divergence and the DF system a $\delta$-function curl at its pole, with uniform and oppositely directed sources elsewhere. By placing a sufficient number of CF and DF CECS at different locations of the ionosphere, one can construct any 2-dimensional vector field from its sources and curls, in accordance with Helmholtz's theorem. The SECS and CECS are related to the Green's function solutions of the curl and divergence operators on the sphere and plane, respectively. Let's consider the curl-free part of the current and define a 2-dimensional Green's function $G_{\mathrm{CF}}$ on a sphere of radius $R$ so that

$\nabla_{p}^{2} G_{\mathrm{CF}}\left(\boldsymbol{r}, \boldsymbol{r}_{p}\right)=\frac{\delta\left(\theta-\theta_{p}\right) \delta\left(\phi-\phi_{p}\right)}{\sin \theta_{p} R^{2}}-\frac{1}{4 \pi R^{2}}$.

According to the theory of Green's functions we can write the current potential $\phi_{J}$ in Eg. (6) as

$\phi_{J}(\boldsymbol{r})=\int_{\text {sphere }} G_{\mathrm{CF}} \nabla_{p}^{2} \phi_{J}\left(\boldsymbol{r}_{p}\right) \mathrm{da}{ }_{p}$,

where $\mathrm{da}_{p}=R^{2} \sin \theta_{p} \mathrm{~d} \theta_{p} \mathrm{~d} \phi_{p}$ is the area element. When we take the gradient of the above equation, the lefthand side is the curl-free part of the current $\boldsymbol{J}_{\text {pot }}$. On the righthand side we can identify the curl-free elementary current system as $\nabla G_{\mathrm{CF}}=\mathbf{J}_{\mathrm{CF}}\left(\boldsymbol{r}^{\prime}\right)$, as this fulfills the definition in Eq. (14) when $\boldsymbol{r}_{p}$ is interpreted as the position of the SECS pole. So we arrive at relation

$\boldsymbol{J}_{\text {pot }}=\int_{\text {sphere }} \frac{\nabla_{p} \cdot \boldsymbol{J}\left(\boldsymbol{r}_{p}\right)}{4 \pi R} \cot \left(\frac{\theta^{\prime}}{2}\right) \hat{\boldsymbol{e}}_{\theta^{\prime}} \mathrm{da}_{p}$,

where the angle $\theta^{\prime}$ and unit vector $\hat{\boldsymbol{e}}_{\theta^{\prime}}$ must be expressed in terms of positions $\boldsymbol{r}, \boldsymbol{r}_{p}$ and unit vectors $\hat{\boldsymbol{e}}_{\theta}, \hat{\boldsymbol{e}}_{\phi}$ (see e.g. Appendix A of Vanhamäki et al., 2003). In completely analogous manner we can derive the relation

$\boldsymbol{J}_{\text {rot }}=\int_{\text {sphere }} \frac{\left(\nabla_{p} \times \boldsymbol{J}\left(\boldsymbol{r}_{p}\right)\right)_{r}}{4 \pi R} \cot \left(\frac{\theta^{\prime}}{2}\right) \hat{\boldsymbol{e}}_{\phi^{\prime}} \mathrm{da}_{p}$,

for the rotational part of the current.

In practical calculations the elementary systems are placed at some discrete grid, and the scaling factors give the divergence and curl of the vector field in the grid cell. For some arbitrary grid cell $k$ we can write the scaling factors as

$$
\begin{aligned}
& I_{k, \mathrm{CF}}=\int_{\text {cell } k} \nabla \cdot \boldsymbol{J} \mathrm{da}_{p}, \\
& I_{k, \mathrm{DF}}=\int_{\text {cell } k}(\nabla \times \boldsymbol{J})_{\mathrm{r}} \mathrm{da}_{p} .
\end{aligned}
$$

This means that the curl and divergence that are in reality distributed over the grid cell are represented by point sources at 


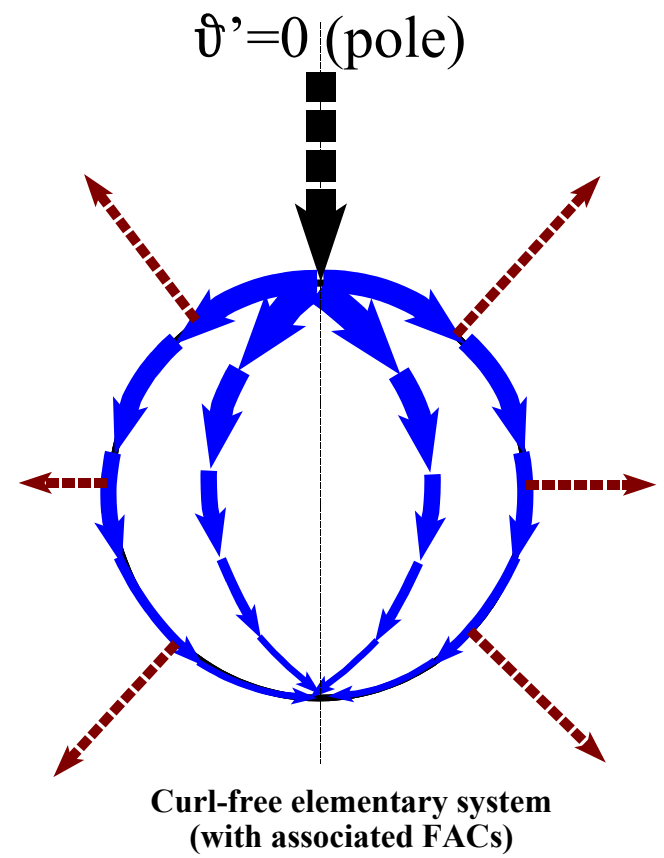

Fig. 2. Spherical elementary current systems (SECS).

the center of the cell. If we want to represent a given vector field $\boldsymbol{J}$ with elementary systems, we may evaluate the integrals in Eqs. (18)-(19) in a suitable grid. However, it is often more practical to evaluate the field explicitly as a sum of elementary systems given in Eqs. (12)-(13) and fit the scaling factors to the given vector field in a least-squares sense. In the fitting process the Laplacian part of the field (see Eq. 10) is represented by outlying elementary systems, so it's important to make the SECS grid somewhat larger than the area of interest.

The main advantage of the elementary systems is that they are intrinsically divided into divergence- and curl-free parts. This division is very natural in ionospheric electrodynamics, where divergent current connects to FAC, rotational current is associated with ground magnetic disturbance, and the inductive electric field is rotational. The number and density of elementary systems used to represent the vector field can be chosen freely, so that higher density of elementary system (better resolution) may be used in areas where there is good data coverage.

Additionally, individual elementary systems are simple enough for analytical treatment. For example, if we use elementary systems to represent currents, and their divergences represent radially inward or outward flowing FAC as in Fig. 2, then the magnetic fields produced by currents in Eqs. (12) and (13) can be calculated analytically in closed form (Amm and Viljanen, 1999).

Specifically, the curl-free elementary system does not cause any magnetic field below the ionosphere, and above it has only an $\hat{\boldsymbol{e}}_{\phi^{\prime}}$ component, as already shown by Fukushima (1976). However, in contrast to Fukushima's paper, this sys-

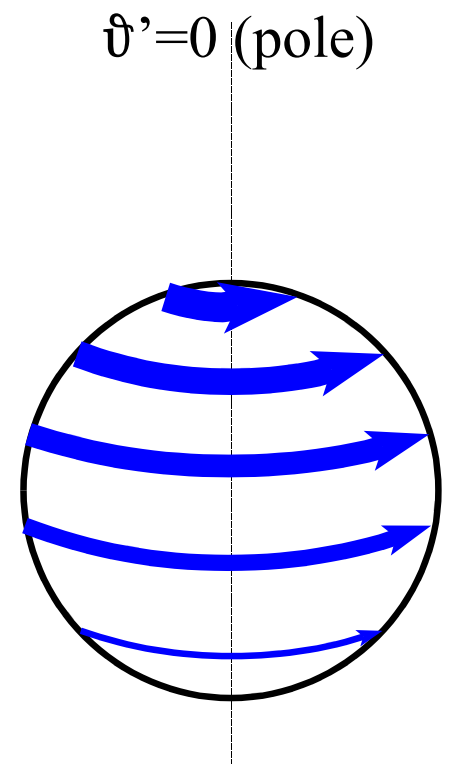

Divergence-free elementary system tem is used as a mere basis function here, and there are no assumptions about ionospheric conductances required, nor about how the currents are constituted by Pedersen and/or Hall currents. In contrast, the divergence-free elementary systems generate magnetic field with $\hat{\boldsymbol{e}}_{r^{\prime}}$ and $\hat{\boldsymbol{e}}_{\theta^{\prime}}$-components both above and below the sphere. Thus, an expansion of a current system in terms of SECS also provides an easy way to calculate the magnetic field of the current system at any point in space. This is utilized for example in analysis of ground magnetic data, as discussed in Sect. 3.3.

For use in situations when the derivate in one horizontal dimension vanishes, here called 1-D situations (see Sect. 7), 1-D SECS have been defined by Vanhamäki et al. (2003) and Juusola et al. (2006). The 1-D variants are obtained by integrating the respective 2-dimensional SECS defined above over a circle at a constant latitude $\theta_{0}$, so that

$$
\begin{aligned}
& \mathbf{J}_{1-\mathrm{D}, \mathrm{CF}}\left(\theta, \theta_{0}\right)=\frac{I_{1-\mathrm{D}, \mathrm{CF}}}{2 R_{\mathrm{I}}} \hat{\boldsymbol{e}}_{\theta}\left\{\begin{array}{ll}
-\tan (\theta / 2), & \theta<\theta_{0} \\
\cot (\theta / 2) & , \theta>\theta_{0}
\end{array},\right. \\
& \mathbf{J}_{1-\mathrm{D}, \mathrm{DF}}\left(\theta, \theta_{0}\right)=\frac{I_{1-\mathrm{D}, \mathrm{DF}}}{2 R_{\mathrm{I}}} \hat{\boldsymbol{e}}_{\phi} \begin{cases}-\tan (\theta / 2), & \theta<\theta_{0} \\
\cot (\theta / 2) & , \theta>\theta_{0}\end{cases}
\end{aligned}
$$

Similar to the general 2-D SECS, the divergence- and curlfree 1-D SECS are basis functions for any continuously differentiable vector field on a sphere, with vanishing derivative in one direction. If they represent a current system, the same properties of the magnetic field of each elementary system as mentioned for the 2-D case hold. 


\section{Determination of equivalent currents}

\subsection{Harmonic analysis}

In the neutral atmosphere between the Earth's surface and the ionosphere, the electric conductivity is vanishingly small. Consequently, in this region the magnetic field can be represented by a potential,

$\boldsymbol{B}=-\nabla \phi_{B}$

where

$\nabla^{2} \phi_{B}=0$.

In harmonic analysis the magnetic potential $\phi_{B}$ is expanded in terms of some basis functions. Typically, spherical harmonics (Chapman and Bartels, 1940) are used in global analysis and spherical cap harmonics (Haines, 1985; revised by Thébault et al., 2006) or plane waves in mesoscale studies (e.g. Richmond and Baumjohann, 1983). The magnitude of different basis functions is then fitted to the magnetic observations, for example by minimizing the residual in the leastsquares sense. Richmond and Baumjohann (1983) present a fitting technique based on the theory of optimal linear estimation, where geophysical constraints on the allowed current systems can be included.

One shortcoming of these spectral methods is that in order to keep the fitting numerically stable, some fixed upper and lower scale lengths must be chosen for the whole analysis area. Variations that are smaller or larger than these scale lengths cannot be modeled accurately. This is a problem if the spatial distribution of magnetometers is highly nonuniform, as the minimum scale length must be chosen according to the sparsest region of the network.

The ionospheric equivalent current is obtained from $\phi_{B}$ by evaluating the magnetic potential at a suitable altitude (typically $\sim 100 \mathrm{~km}$ ) above the Earth's surface and calculating the jump condition

$$
\Delta \boldsymbol{B}_{\perp}=-\mu_{0} \hat{\boldsymbol{e}}_{\mathrm{r}} \times \boldsymbol{J}_{\text {eq,ion }}
$$

over the assumed current sheet. Further details are given e.g. by Haines and Torta (1994).

\subsection{General comments about $J_{\text {eq }}$ calculation}

It should be noted that the horizontal component of the ground magnetic disturbance field can be explained by using just external (ionospheric) equivalent currents, even if part of the disturbance is created by internal currents flowing in the conducting Earth. This is clear from the expansion of the potential $\phi_{B}$ in terms of spherical harmonic or spherical cap harmonic functions (Untiedt and Baumjohann, 1993). The internal and external contributions to the magnetic disturbance field can be separated by using all 3 components of the magnetic field. Further details are given e.g. in chapter 20 of Chapman and Bartels (1940) and in Haines and Torta (1994).
Except for some very dynamical situations (e.g. Tanskanen et al., 2001), the contribution from internal currents can normally be neglected and $\boldsymbol{J}_{\text {eq,ion }}$ is determined using only the horizontal part of the ground magnetic disturbance $\boldsymbol{B}_{\mathrm{G}, \perp}$. The justification is that the internal part of $\boldsymbol{B}_{\mathrm{G}, \perp}$ is usually spatially quite smooth when compared to the external part, so it adds only a rather homogeneous background to the equivalent current (see e.g. Sect. 2.3 of Untiedt and Baumjohann, 1993, and references therein). On the other hand, the radial part of $\boldsymbol{B}_{\mathrm{G}}$ is heavily affected by internal induced currents and it is also very sensitive to local anomalies in the Earth's conductivity (e.g. the coast effect, see Parkinson and Jones, 1979). Consequently, it is more difficult to separate the observed $\boldsymbol{B}_{\mathrm{G}}$ into internal and external parts, than to represent just the horizontal part of the disturbance in terms of $\boldsymbol{J}_{\text {eq,ion }}$. Further, in some techniques, like the method of characteristics, only $\left(\nabla \times J_{\text {eq,ion }}\right)_{r}$ is actually used, so that any nearly uniform background current vanishes to a good approximation.

\subsection{SECS analysis}

Amm and Viljanen (1999) introduced a SECS-based method for determining the ionospheric equivalent current. In this method several divergence-free elementary systems are placed at a 2-dimensional horizontal grid in the ionosphere, and their scaling factors are chosen so that the observed magnetic disturbances are matched as closely as possible in the least squares sense.

In the SECS analysis the horizontal components of measured $\boldsymbol{B}_{\mathrm{G}}$ at locations $\boldsymbol{r}_{n}=\left(R_{\mathrm{E}}, \theta_{n}, \phi_{n}\right)$ are collected in one vector

$\mathfrak{B}_{\perp}=\left[B_{\mathrm{X}}\left(\boldsymbol{r}_{1}\right) B_{\mathrm{y}}\left(\boldsymbol{r}_{1}\right) B_{\mathrm{X}}\left(\boldsymbol{r}_{2}\right) \ldots\right]^{T}$

while the unknown scaling factors of the DF SECS located at $\boldsymbol{r}_{k}^{\mathrm{el}}=\left(R_{\mathrm{I}}, \theta_{k}^{\mathrm{el}}, \phi_{k}^{\mathrm{el}}\right)$ are collected in another vector

$\mathfrak{I}_{\mathrm{DF}}=\left[I_{\mathrm{DF}}\left(\boldsymbol{r}_{1}^{\mathrm{el}}\right) I_{\mathrm{DF}}\left(\boldsymbol{r}_{2}^{\mathrm{el}}\right) I_{\mathrm{DF}}\left(\boldsymbol{r}_{3}^{\mathrm{el}}\right) \ldots\right]^{T}$.

These vectors are connected by a transfer matrix $\mathbf{T}$, so that

$\mathfrak{B}_{\perp}=\mathbf{T} \cdot \mathfrak{I}_{\mathrm{DF}}$.

The components of transfer matrix $\mathbf{T}$ give the magnetic field caused by each individual unit SECS at the magnetometer sites, and is therefore known and depends only on geometry. For example, $T_{2,4}$ gives the y-component (East) of $\boldsymbol{B}_{\mathrm{G}}$ at $\boldsymbol{r}_{1}$ caused by the SECS centered at $\boldsymbol{r}_{4}^{\text {el }}$. Details how to calculate the matrix $\mathbf{T}$ and how to invert Eq. (27) for the unknown scaling factors $\mathfrak{I}_{\mathrm{DF}}$ using truncated singular value decomposition are given by Amm and Viljanen (1999) and Pulkkinen et al. (2003b). Once the scaling factors are known, the actual equivalent current $\boldsymbol{J}_{\text {eq,ion }}$ can be calculated using Eq. (13) for each individual DF SECS separately.

Amm and Viljanen (1999) tested the SECS-based calculation of equivalent current in a simulated case of a Cowling channel. They also compared the SECS-based method 


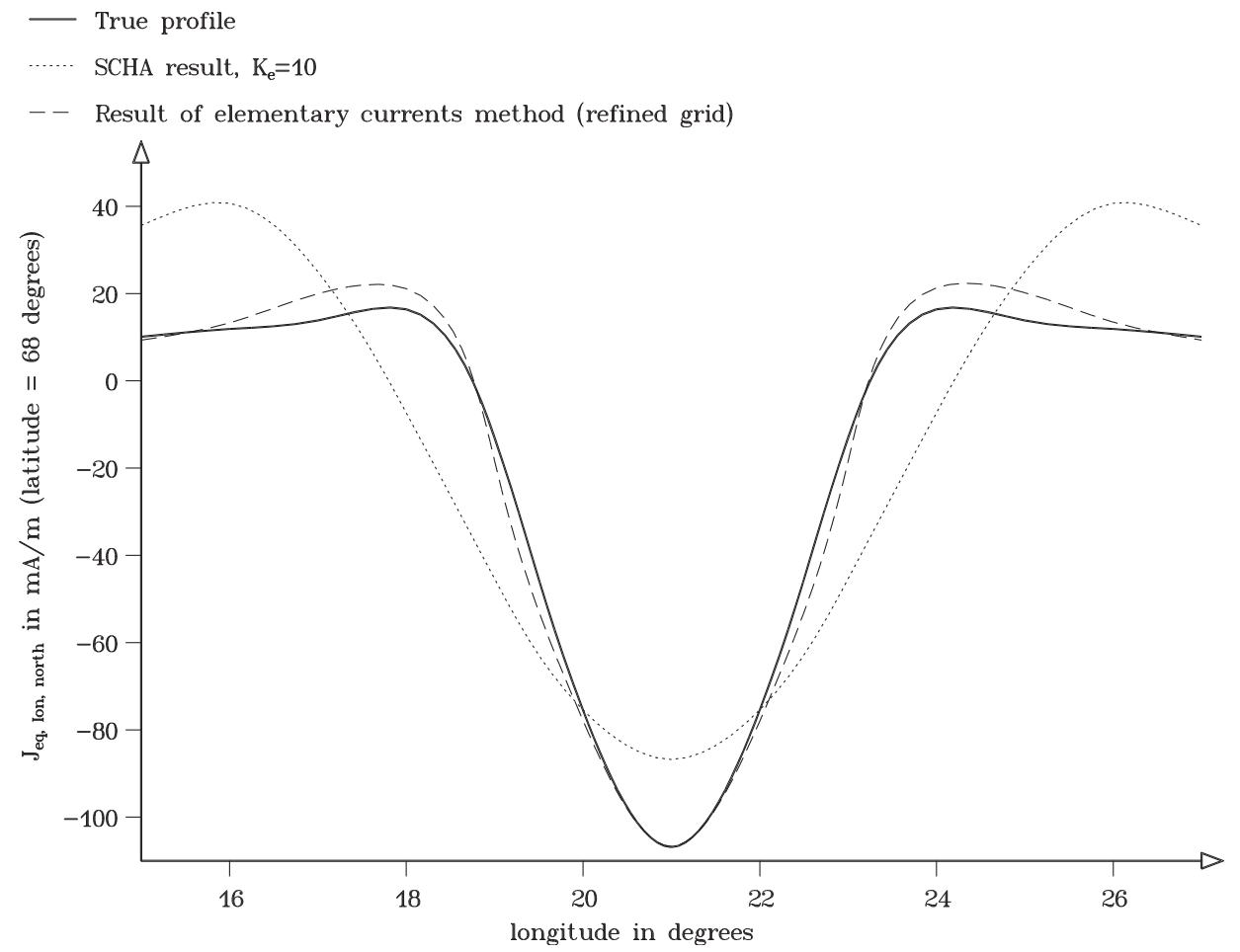

Fig. 3. Longitudinal profile of the north component of the ionospheric equivalent currents in the test model. Solid line: true profile; Dotted line: SCHA upward continuation result with up to 10th order basis functions; Broken line: SECS method upward continuation result. Reproduced from Fig. 6 of Amm and Viljanen (1999).

against spherical cap harmonic analysis (SCHA) developed by Haines (1985). The results of the comparison are shown in Fig. 3. The SECS-based method gives more accurate results, partly because there is no need to specify any fixed limit for the smallest resolved wavelength like in the SCHA. In the SECS-analysis the poles of elementary systems can freely be placed where they are most suitable with respect to the density of measurements.

The SECS-based calculation of equivalent current has been further tested by Pulkkinen et al. (2003a), while Pulkkinen et al. (2003b) extended the method so that the ground magnetic disturbance may be separated into internal and external parts by using 2 layers of DF SECS, one in the ionosphere and the other inside the Earth. There is also a 1dimensional variant of the method, as indicated in Table 1, developed by Vanhamäki et al. (2003) and Juusola et al. (2006). This is discussed in Sect. 7 together with other 1dimensional analysis methods.

\section{Current as the primary output}

Amm (2001) developed the elementary current method (see Table 1) for calculating the actual (not equivalent) ionospheric current $\boldsymbol{J}$ using the ground magnetic field $\boldsymbol{B}_{\mathrm{G}}$ and field-aligned current $j_{\|}$measured by a fleet of satellites as input data. The ionospheric current is written as a sum of its curl- and divergence-free parts as in Eq. (10),

$\boldsymbol{J}=\boldsymbol{J}_{\text {pot }}+\boldsymbol{J}_{\text {rot }}$

The divergence-free part $\boldsymbol{J}_{\text {rot }}$ is obtained from ground magnetic field, as discussed in the previous section, and $\boldsymbol{J}_{\text {pot }}$ can be integrated from the FAC.

Both curl- and divergence-free parts of $\boldsymbol{J}$ are straightforwardly obtained using the elementary current systems, discussed in Sect. 2.3, hence the name of the method used in Table 1. SECS-based analysis of the ground magnetic data is discussed in Sect. 3.3. The field-aligned current $j_{\|}$can be directly integrated using Eqs. (4), (18) and (12), thus giving the curl-free part of the current. If data is available globally, $\boldsymbol{J}$ is uniquely determined. However, in mesoscale studies the solution is not unique, for a Laplacian field with zero curl and divergence inside the analysis area can be added to $\boldsymbol{J}$, as in Eq. (10).

Ground magnetic data is readily available from different magnetometer networks, but FAC measurements suitable for event analysis are harder to obtain. A fleet of nearby located satellites (such as Cluster or Swarm) can provide multi-point measurements of FAC over a limited region. However, observations spanning several minutes have to be combined, so that a sufficient number of data points to infer 2-dimensional FAC distribution is obtained. Large scale FAC distribution 
can be estimated using engineering magnetometer data from the Iridium satellite constellation (Anderson et al., 2000; Green et al., 2006), although at a limited spatial and temporal resolution.

In the elementary current method the ionospheric current is obtained directly from the equivalent currents (derived from the ground magnetic field data) and the FAC distribution (derived from the satellite magnetic field data), without any assumed parameters. If also measurements of the electric field are available (e.g. from radars), the ionospheric conductances are obtained from Eq. (3). Amm (2001) studied the applicability of the elementary current method using a simulated passage of the Cluster spacecraft over the MIRACLE network illustrated in Fig. 1. The results shown in Fig. 4 demonstrate that the ionospheric current, as well as the Hall and Pedersen conductances can be reconstructed by the method to a good accuracy.

Green et al. (2007) estimated the large scale ionospheric conductance distribution by combining ground and satellitebased magnetic measurements with $\boldsymbol{E}$ obtained from SuperDARN and DMSP satellite. Instead of using elementary systems, Green et al. (2007) derived the ionospheric current by using two potentials, as in Eq. (6). The potentials were expanded in terms of spherical cap harmonics (discussed in Sect. 3.1) and fitted to the magnetic data in a $40^{\circ}$ cap. The results obtained by Green et al. (2007) in an event study agree with statistical models, although sparse data coverage resulted in significant uncertainties.

One possible addition to the existing analysis methods may be obtained, if we assume that the conductance ratio $\alpha=\Sigma_{\mathrm{H}} / \Sigma_{\mathrm{P}}$, instead of the electric field, is known in addition to $\boldsymbol{J}$. This possibility is discussed in connection with the Method of Characteristics in Sect. 6.

\section{Electric field as the primary output}

In Table 1 we have included two methods that give the ionospheric electric field as the primary output. The KRMmethod (named after Kamide, Richmond and Matsushita) was introduced by Kamide et al. (1981) and takes ground magnetic field $\boldsymbol{B}_{\mathrm{G}}$ (or equivalent current) and ionospheric conductances as input data. In the 4 th $\operatorname{method}^{1}$ the field aligned current $j_{\|}$is used instead of $\boldsymbol{B}_{\mathrm{G}}$.

In a sense, also the AMIE-method (Assimilative Mapping of Ionospheric Electrodynamics, introduced by Richmond and Kamide, 1988) falls into this category, as it gives the ionospheric electric potential as the main output. AMIE is a data assimilation procedure, essentially based on Optimal Interpolation theory (e.g. Matsuo et al., 2005). It assimilates measured electric fields, ionospheric currents, and magnetic field perturbations into a statistical model of the electric potential, assuming that the the ionospheric conductances are

\footnotetext{
${ }^{1}$ As far as we are aware, there is no commonly used name for this method.
}

given as input data. However, AMIE is not really suitable for mesoscale event studies, as it is designed for global-scale analysis, and relies quite heavily on statistical models of the electric potential and conductances. For further discussion about AMIE and examples of application see e.g. Knipp et al. (1993) or Richmond et al. (1998), and references therein.

\subsection{KRM and FAC-based electric field calculation}

The curl and divergence of the Ohm's law are written out in Eqs. (8)-(9). The divergence of the horizontal current is directly connected to the FAC, as in Eq. (4). The curl of $\boldsymbol{J}$ can be calculated from the ionospheric equivalent current, as discussed in Sect. 2.2,

$(\nabla \times \boldsymbol{J})_{\mathrm{r}}=\left(\nabla \times \boldsymbol{J}_{\text {eq,ion }}\right)_{\mathrm{r}}$,

It is usually assumed that the ionospheric electric field is a potential field (inductive effects are neglected), so that

$\boldsymbol{E}=-\nabla \phi_{E}$.

While not always exactly true (see Sect. 8), Eq. (30) is usually a good approximation and simplifies the theory considerably.

Putting Eqs. (8)-(9) and (29)-(30) together, we get two second order partial differential equations for the electric potential $\phi_{E}$,

$$
\begin{aligned}
& \Sigma_{\mathrm{H}} \nabla^{2} \phi_{E}+\nabla \Sigma_{\mathrm{H}} \cdot \nabla \phi_{E}-\left(\nabla \Sigma_{\mathrm{P}} \times \nabla \phi_{E}\right)_{\mathrm{r}}=\left(\nabla \times J_{\text {eq,ion }}\right)_{\mathrm{r}},(31) \\
& \Sigma_{\mathrm{P}} \nabla^{2} \phi_{E}+\nabla \Sigma_{\mathrm{P}} \cdot \nabla \phi_{E}-\left(\nabla \Sigma_{\mathrm{H}} \times \nabla \phi_{E}\right)_{\mathrm{r}}=-j_{\|}
\end{aligned}
$$

These equations can be solved for the electric potential, once the ionospheric conductances, FAC or $\boldsymbol{J}_{\text {eq,ion }}$ and the boundary condition for $\phi_{E}$ are specified.

Equation (31) forms the basis of the KRM method, which has been extensively applied in event studies as well as in statistical investigations (see e.g. Ahn et al., 1995; Kamide et al., 1996; Glassmeier, 1987; Untiedt and Baumjohann, 1993, and references therein). On the other hand, Eq. (32) is mainly used in statistical studies, where global FAC distributions are obtained by integrating satellite observations of $j_{\|}$over multiple orbits (see e.g. Sect. 2.4.3 of Untiedt and Baumjohann, 1993, and references therein). Also global MHD simulations usually solve the ionospheric part of the simulation by giving conductances and FAC as output from the magnetosphere, and then map the resulting potential electric field back to the magnetosphere (e.g. Janhunen, 1998).

Ground magnetic measurements, and therefore also

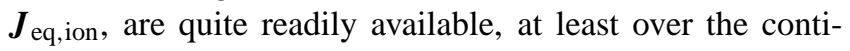
nents. However, it is very hard to measure FAC distribution over an extended region with sufficient spatial and temporal resolution that Eq. (32) could be used in an event study, although estimates based on Iridium satellite data (e.g. Anderson et al., 2000) might be suitable.

Probably the greatest uncertainties in solving Eqs. (31) and (32) are caused by the input conductance distributions, as 


\section{Model}
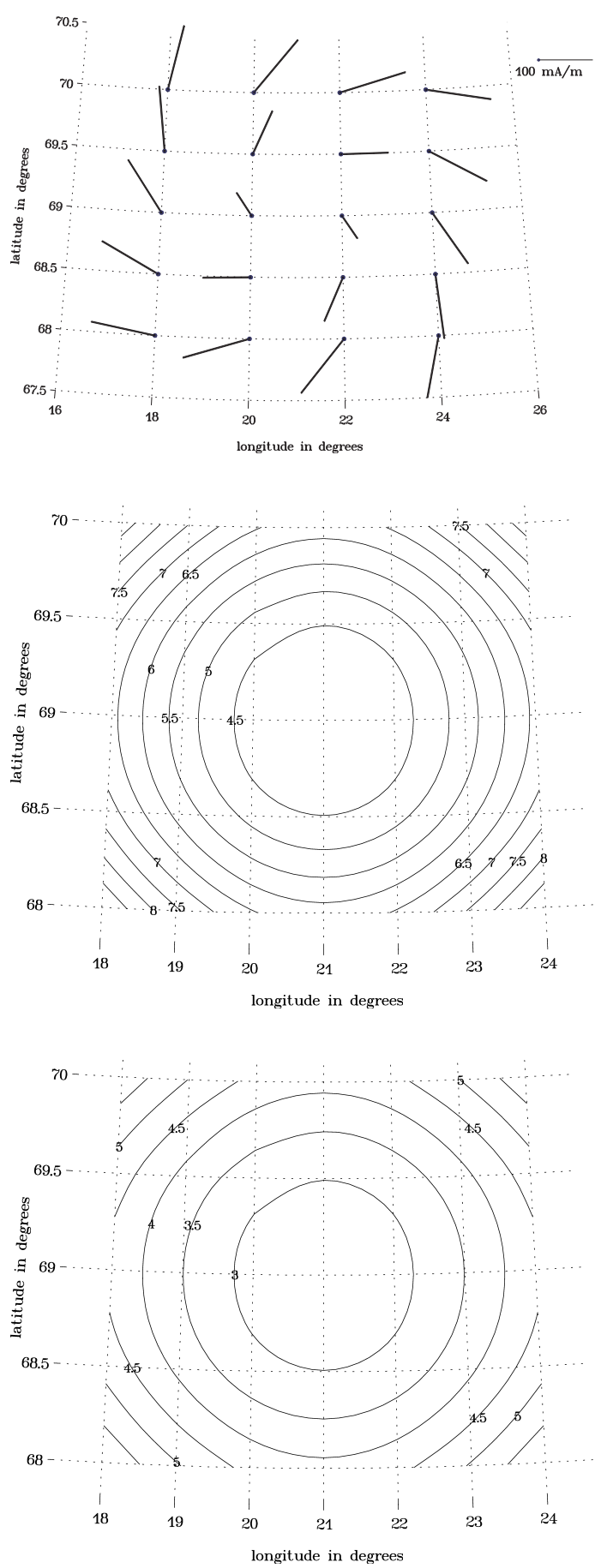

Reconstruction
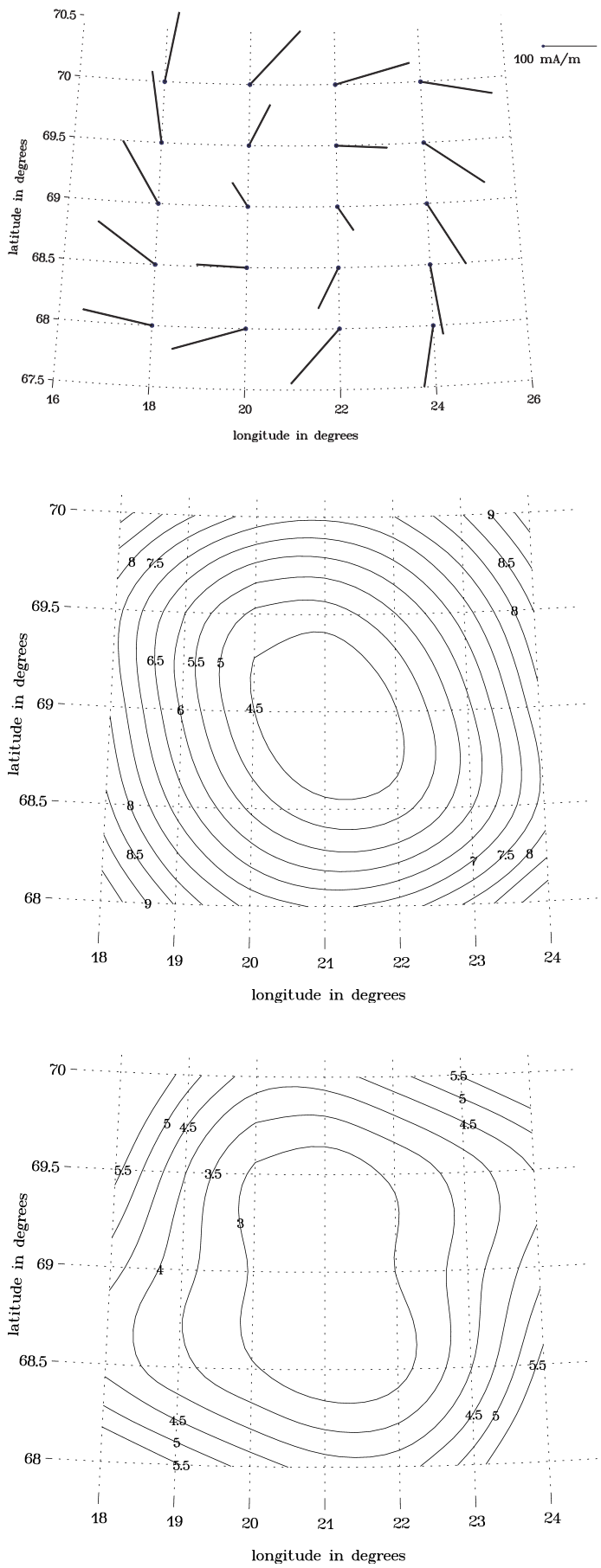

Fig. 4. Original model (left) and results of the elementary current method (right). From top to bottom: True ionospheric currents, Hall conductance (in siemens) and Pedersen conductance (in siemens). Reproduced from Figs. 3 and 5 of Amm (2001). 
demonstrated by Murison et al. (1985) for the KRM method. Two-dimensional ionospheric conductance distributions are quite difficult to obtain from direct measurements, but estimates may be derived from satellite or all-sky camera images and riometer data (e.g., Lummerzheim et al., 1991; Janhunen, 2001; Aksnes et al., 2005; Senior et al., 2008). However, these estimates rely on semi-empirical formulas that first relate optical emissions to the flux and characteristic energy of the precipitating electrons, which are then used to estimate the height-integrated conductances. Statistical models (e.g., Fuller-Rowell and Evans, 1987) are also available and a rough conductance estimates may be derived from the ground magnetic data (Ahn et al., 1998), but these are less suitable for event analysis.

If Eqs. (31) or (32) are solved globally, we only have to fix the zero level of $\phi_{E}$. Also in semi-global studies, that cover either the northern or southern auroral regions, boundary conditions for the electric potential are not problematic, as they have to be specified only at the mid-latitudes, where the electric field is rather small in any case. However, in mesoscale studies boundary conditions play an important role.

\subsection{Local solution}

Murison et al. (1985) solved Eq. (31) in a mesoscale study, where the Harang discontinuity was present over northern Scandinavia. They found that the electric field, and consequently also the currents, strongly depend on the boundary conditions that are imposed on $\phi_{E}$ at the boundaries of the analysis area. This severely limits the use of the KRM method in regional studies. Also the FAC-based method is affected by boundary conditions in a similar manner.

Kamide et al. (2003) developed a local variant of the KRM method, where the KRM equation is solved in areas of good data coverage and the required boundary conditions are obtained using the AMIE technique (Richmond and Kamide, 1988). This allows use of the local KRM in a straightforward manner, for example in real-time space weather monitoring. However, it should be kept in mind that in absence of global data coverage AMIE gives results that are mostly based on statistical models. Therefore the obtained boundary conditions, and also the KRM solution, may not be accurate enough for studying specific events in detail.

More recently Vanhamäki and Amm (2007) introduced a different, SECS-based approach of solving the local KRM problem. Instead of directly solving the electric field, Vanhamäki and Amm (2007) try to find such a curl-free current system $\boldsymbol{J}_{\mathrm{CF}}$, that together with the equivalent current $\boldsymbol{J}_{\text {eq,ion }}$ and the given conductances, the electric field becomes a potential field. Vanhamäki (2010) generalized this approach so that either $\boldsymbol{J}_{\text {eq,ion }}$ or FAC can be used as input. Also spherical geometry, tilted magnetic field lines and inductive effects were taken into account, so that the method can be used in global studies as well. A brief review of the method is given below.

\subsection{Solution with elementary systems}

With SECS we can calculate the horizontal current from its curl and divergence, as

$\mathfrak{J}=\mathbf{M}_{1} \cdot \mathfrak{I}_{\mathrm{CF}}+\mathbf{M}_{2} \cdot \mathfrak{I}_{\mathrm{DF}}$

The vector $\mathfrak{J}$ contains the $\theta$ - and $\phi$-components of $\boldsymbol{J}$ at the grid points $\boldsymbol{r}_{n}=\left(R_{\mathrm{I}}, \theta_{n}, \phi_{n}\right)$,

$\mathfrak{J}=\left[\begin{array}{llll}J_{\theta}\left(\boldsymbol{r}_{1}\right) & J_{\phi}\left(\boldsymbol{r}_{1}\right) & J_{\theta}\left(\boldsymbol{r}_{2}\right) \ldots\end{array}\right]^{T}$,

while the vectors $\mathfrak{I}_{\mathrm{CF}}$ and $\mathfrak{I}_{\mathrm{DF}}$ contain the scaling factors of the CF and DF SECS at grid points $\boldsymbol{r}^{\mathrm{el}}$, respectively

$$
\begin{aligned}
& \mathfrak{I}_{\mathrm{CF}}=\left[I_{\mathrm{CF}}\left(\boldsymbol{r}_{1}^{\mathrm{el}}\right) I_{\mathrm{CF}}\left(\boldsymbol{r}_{2}^{\mathrm{el}}\right) I_{\mathrm{CF}}\left(\boldsymbol{r}_{3}^{\mathrm{el}}\right) \ldots\right]^{T}, \\
& \mathfrak{I}_{\mathrm{DF}}=\left[I_{\mathrm{DF}}\left(\boldsymbol{r}_{1}^{\mathrm{el}}\right) I_{\mathrm{DF}}\left(\boldsymbol{r}_{2}^{\mathrm{el}}\right) I_{\mathrm{DF}}\left(\boldsymbol{r}_{3}^{\mathrm{el}}\right) \ldots\right]^{T},
\end{aligned}
$$

Here $I_{\mathrm{DF}}\left(\boldsymbol{r}^{\mathrm{el}}\right)$ and $I_{\mathrm{CF}}\left(\boldsymbol{r}^{\mathrm{el}}\right)$ should be interpreted as the average divergence and curl of $\boldsymbol{J}$ over the grid cells, as in Eqs. (18) and (19). The components of the transfer matrices $\mathbf{M}_{1,2}$ can be calculated using Eqs. (12) and (13), as explained in detail by Vanhamäki (2010).

The electric field can be solved from Ohm's law as

$$
\boldsymbol{E}=\left(\Sigma_{\mathrm{P}} \boldsymbol{J}-\Sigma_{\mathrm{H}} \hat{\boldsymbol{e}}_{z} \times \boldsymbol{J}\right) /\left(\Sigma_{\mathrm{P}}^{2}+\Sigma_{\mathrm{H}}^{2}\right) .
$$

For simplicity we assume here a vertical (radial) background magnetic field. The necessary modifications for tilted field lines have been included by Vanhamäki (2010).

The curl and divergence of the inverted Ohm's law give us two relations between the electric field and current. In this case we need only the curl of $\boldsymbol{E}$, which can be written in terms of elementary systems as

$\mathfrak{c u r l e}=\mathbf{L}_{1} \cdot \mathfrak{I}_{\mathrm{CF}}+\mathbf{L}_{2} \cdot \mathfrak{I}_{\mathrm{DF}}$.

The vector curle contains $r$-component of the curl of the electric field at the grid points $\boldsymbol{r}^{\mathrm{el}}$ and is analogous to the vector $\mathfrak{J}$ defined above. The matrices $\mathbf{L}_{1,2}$ can be constructed using the previously defined matrices $\mathbf{M}_{1,2}$ and the inverted Ohm's law, as outlined by Vanhamäki (2010).

If we use the potential approximation $(\nabla \times \boldsymbol{E})_{r}=0$, we get a simple relation

$\mathbf{L}_{1} \cdot \mathfrak{I}_{\mathrm{CF}}+\mathbf{L}_{2} \cdot \mathfrak{I}_{\mathrm{DF}}=0$.

This can be solved for either $\mathfrak{I}_{\mathrm{CF}}$ (FAC) or $\mathfrak{I}_{\mathrm{DF}}$ (equivalent current), depending on which one is given as the input. Ionospheric induction can be included by relating $\mathfrak{c u r l \mathfrak { E }}$ to $\mathfrak{I}_{\mathrm{CF}}$ and $\mathfrak{I}_{\mathrm{DF}}$ via Faraday's and Ampere's laws. This is discussed in more detail in Sect. 8.2.

One of the advantages in the SECS-based approach is the automatic inclusion of boundary conditions in mesoscale 
studies. As the vector fields are constructed from divergences and curls, the natural and implicitly included boundary condition is to assume that outside the analysis area the vector fields are source-free. This is probably not a totally correct assumption in most situations, but it is a very convenient one and has been proven to work quite well in practice (Vanhamäki and Amm, 2007).

It should be noticed that in the traditional KRM method only the local quantity $\left(\nabla \times \boldsymbol{J}_{\text {eq,ion }}\right)_{\mathrm{r}}$ enters the differential equation, even if $\boldsymbol{J}_{\text {eq,ion }}$ were known in a larger area than $\Sigma_{\mathrm{H}}$ and $\Sigma_{\mathrm{P}}$, which is often the case in mesoscale studies. In the SECS-based approach the whole vector $\boldsymbol{J}_{\text {eq,ion }}$ is used in constructing the matrices $\mathbf{M}_{1,2}$, so that all the information available from the ground magnetic field is taken into account. This difference may have a significant impact in mesoscale studies, both for the KRM and FAC-based methods.

Vanhamäki and Amm (2007) tested the SECS-based KRM method and compared the results against the traditional KRM solutions using several realistic models of typical mesoscale phenomena in the auroral ionosphere. In the SECS-based KRM results the average error over the whole analysis area is typically around $20-40 \%$, whereas the errors in the traditional KRM results are significantly larger. Figure 5 shows one example, where the analysis method is applied to a databased model of a westward traveling surge. The input model used in the analysis consists of Hall and Pedersen conduc-

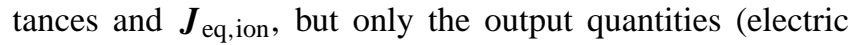
field, horizontal current and FAC) are shown in Fig. 5, together with their deviations from the original model. Apart from some errors near the boundaries, the results are reasonably accurate.

\section{Conductances as the primary output}

A weak point of the techniques discussed in the previous section is that both ionospheric conductances need to be input quantities. Direct conductance measurements are difficult to obtain, and on a mesoscale area with a time resolution of the order of seconds such measurements are unavailable with the present day instrumentation. As discussed before, the use of statistical conductance models, or the estimation of conductances from ground-based or satellite optical data both involve a substantial number of intrinsic approximations. In case of statistical models, smaller scale variations in the actual conductance distribution may not be well represented by the statistical prediction, or it even might not represent the actual conductance distribution very well at all.

For the methods discussed in this section, only the Hall to Pedersen conductance ratio $\alpha=\Sigma_{\mathrm{H}} / \Sigma_{\mathrm{P}}$ is needed as an input parameter, and the individual conductance distributions are the primary output of the techniques. Not only does this remove one degree of freedom from the total amount of assumptions needed (cf. Table 1), but it also has the advantage that $\alpha$ is more accessible for an estimation by ground-based data sets than the individual conductances themselves. This has been shown by Lester et al. (1996), and recently Juusola et al. (2007) obtained well-defined statistical relations between $\alpha$ and the magnitude of the zonal component of the ionospheric equivalent currents.

On the other hand, these methods require the ionospheric electric field distribution as input. On a mesoscale area, such data are at present only available from ionospheric coherent scatter radar systems such as STARE (Greenwald et al., 1978, discontinued in 2005) or SuperDARN (Greenwald et al., 1995). The actual availability of data depends on whether or not the ionospheric condition is favorable for the radars to receive a signal scattered back from ionospheric irregularities (e.g. Milan et al., 1997). In the future, with improving technologies and increasing numbers of measurement devices, also incoherent scatter radar systems such as AMISR (Nicolls and Heinselman, 2007) or the planned EISCAT 3-D are expected to be able to provide data sets of the ionospheric electric field on an extended area.

The technique for solving first the ionospheric Hall conductance from spatial measurements of the ground magnetic field, the ionospheric electric field, and an assumption for $\alpha$ is called "method of characteristics". It has first been developed by Inhester et al. (1992) in Cartesian coordinates, then been extensively tested with modeled data sets by Amm (1995), and finally been defined for a spherical geometry by Amm (1998). In this original version of the method, in the first step, the ground magnetic field data are used to calculate ionospheric equivalent currents, as described in Sect. 3.3. Therefore, this version which solely relies on ground-based data is also called the " $\boldsymbol{J}_{\text {eq }}$-based" version of the method of characteristics. Later on, Amm (2002) showed that if spatial measurements of field-aligned currents are available from satellite data, this data can be used to replace the ionospheric equivalent currents as input. This second version of the method of characteristics is thus called the "FAC-based" version. Since both versions are mathematically completely analogous and only use different parameters, in the following we present the $J_{\text {eq }}$-based version of the technique, and only briefly mention the differences of the FAC-based version thereafter. In Sect. 9.1 we outline two possible extensions of the method of characteristics, so that more diverse input data sets could be analyzed.

Using Ohm's law (3), the current continuity relation (4) and the calculation of ionospheric equivalent currents from the ground magnetic field disturbance (Sect. 3.3), and by defining a vector field $\boldsymbol{V}$ as

$$
\boldsymbol{V}=\boldsymbol{E}-\left(\boldsymbol{E} \times \hat{\boldsymbol{e}}_{\mathrm{r}}\right) / \alpha
$$

and two scalar fields $C$ and $D$ as

$\boldsymbol{C}=\nabla \cdot \boldsymbol{V}$,

$D=-\left(\nabla \times J_{\text {eq,ion }}\right)_{\mathrm{r}}$ 

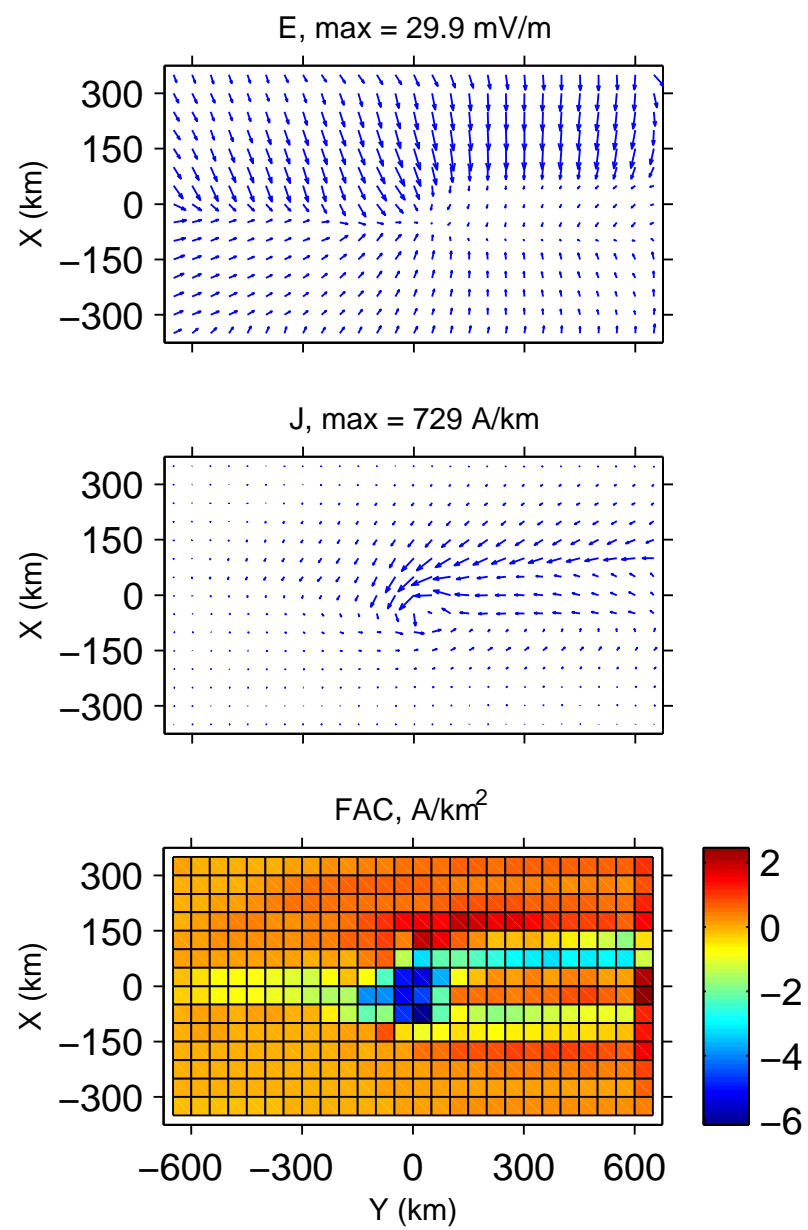
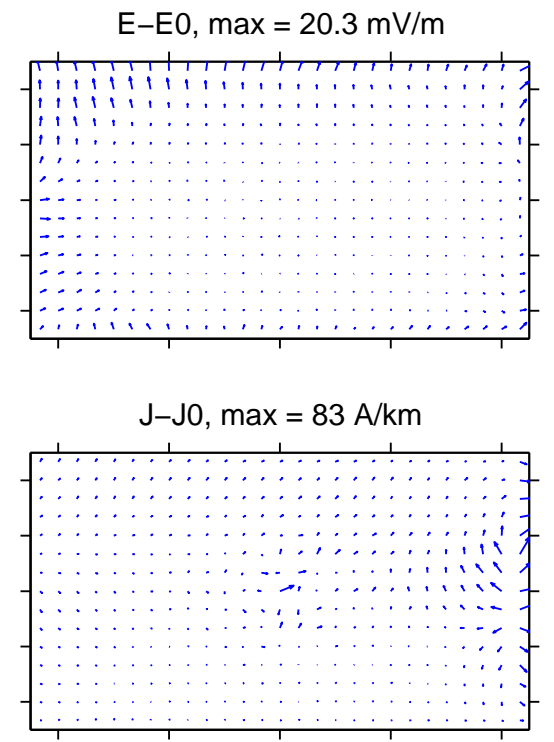

FAC-FACO, A/km²

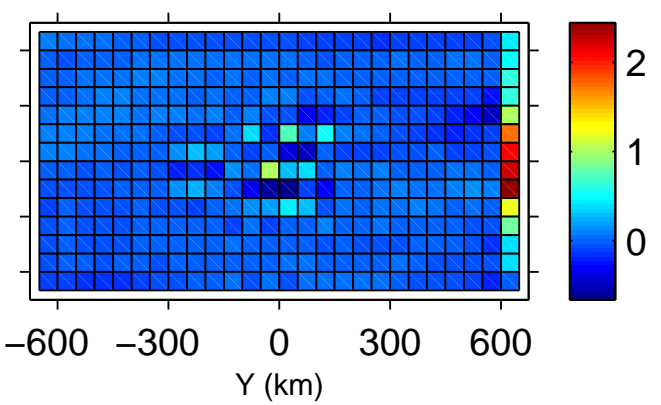

Fig. 5. Example where the elementary system -based local KRM method is applied to an empirical model of a westward traveling surge. Lefthand panels show the electric field, horizontal current and FAC obtained with the SECS-based KRM method, while the righthand panels show the difference between the solution and the original model. Adapted from Fig. 10 of Vanhamäki and Amm (2007).

we arrive at the following first-order partial differential equation:

$\frac{1}{R_{\mathrm{I}}} \frac{\partial \Sigma_{\mathrm{H}}}{\partial \theta} V_{\theta}+\frac{1}{R_{\mathrm{I}} \sin \theta} \frac{\partial \Sigma_{\mathrm{H}}}{\partial \phi} V_{\phi}+C \Sigma_{\mathrm{H}}=D$

This can be solved for $\Sigma_{\mathrm{H}}$, since $V, C$, and $D$ are known from the input data and from the assumption of $\alpha . R_{\mathrm{I}}$ is the distance of the ionospheric current sheet from the Earth's center, typically approximated as $6471 \mathrm{~km}$. The main idea of the method of characteristics is to split up Eq. (43) into two ordinary first-order differential equations, and then solve $\Sigma_{\mathrm{H}}$ by integrating the first of these equations along the characteristics of Eq. (43), which are given by the second equation. The solution for $\Sigma_{\mathrm{H}}$ along each characteristic $\boldsymbol{r}(l)$ is then given by

$\Sigma_{\mathrm{H}}(\boldsymbol{r}(l))=\Sigma_{\mathrm{H}}\left(\boldsymbol{r}_{0}\right) e^{-I(0, l)}+\int_{0}^{l} \frac{D\left(\boldsymbol{r}\left(l^{\prime}\right)\right) e^{-I\left(l^{\prime}, l\right)}}{\left|\boldsymbol{V}\left(\boldsymbol{r}\left(l^{\prime}\right)\right)\right|} \mathrm{d} l^{\prime}$, with

$I\left(l^{\prime}, l\right)=\int_{l^{\prime}}^{l} \frac{C\left(\boldsymbol{r}\left(l^{\prime \prime}\right)\right)}{\left|\boldsymbol{V}\left(\boldsymbol{r}\left(l^{\prime \prime}\right)\right)\right|} \mathrm{d} l^{\prime \prime}$.

The characteristics $\boldsymbol{r}(l)$, with $l$ being the geometric path lenght along the characteristic, are defined by

$\frac{\mathrm{d}}{\mathrm{d} l} \boldsymbol{r}(l)=\frac{V_{\theta}(\boldsymbol{r}(l)) \sin \theta \hat{\boldsymbol{e}}_{\theta}+V_{\phi}(\boldsymbol{r}(l)) \hat{\boldsymbol{e}}_{\phi}}{R_{\mathrm{I}} \sin \theta|\boldsymbol{V}(\boldsymbol{r}(l))|}$.

The solution of Eq. (45) consists of two terms, in the first of which an unknown boundary value $\Sigma_{\mathrm{H}}\left(\boldsymbol{r}_{0}\right)$ occurs, while the second term is fully determined by the input quantities. The influence of the unknown boundary value decreases exponentially with positive $I(0, l)$, and typically it becomes insignificant after a short distance along the characteristic. In case that $I(0, l)$ is predominantly negative along a characteristic, the direction of integration is reversed, which changes the sign of $I(0, l)$, in order to again obtain a decreasing influence of the unknown boundary value. Since every characteristic intersects the boundary of the region under study 

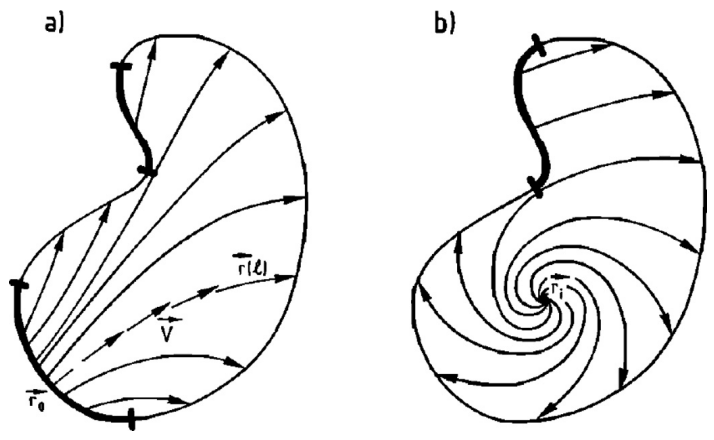

Fig. 6. Sketch illustrating the relation between the tangent field $\boldsymbol{V}$, the characteristic $\boldsymbol{r}(l)$ generated by $\boldsymbol{V}$, and the two types of boundaries of the domain, the "influencing" and "dependent" boundaries. The boundary curve is drawn as a heavy line where it is of the "influencing" type. Note how in (b) the presence of a singular point changes the characteristics and diminishes the "influencing" part on the boundary curve as compared to (a), the case without a singularity. Reproduced from Inhester et al. (1992).

twice, boundary values are needed at most for $50 \%$ of the total boundary. Typically, due to the positive divergence of the characteristics in the direction of integration, as is assured by a positive $I(0, l)$, the part of the boundary for which boundary values are needed is far less than half of the total boundary (see Fig. 6, left panel). A special case are characteristics that end into a "singular point" (Fig. 6, right panel). At these points, $\Sigma_{\mathrm{H}}$ can be directly calculated from the data, and thus no boundary values are needed for the integration along such characteristics. It is noted that if the analysis area is the whole northern or southern auroral zone, and if the electric field has a zero potential outside of this zone, then every characteristic will end in a singular point. Therefore, in this case no boundary conditions are needed at all.

With the resulting distribution of $\Sigma_{\mathrm{H}}$ from the successive application of Eq. (44), together with the input distributions, the remaining set of the ionospheric electrodynamic parameters can then easily be calculated using Eqs. (3) and (4).

As mentioned above, the FAC-based method of characteristics is mathematically completely analogous to the $\boldsymbol{J}_{\text {eq }}$ -based version. Only the following replacements need to be made:

$\boldsymbol{V} \rightarrow \boldsymbol{W}=\boldsymbol{E} / \alpha+\boldsymbol{E} \times \hat{\boldsymbol{e}}_{\mathrm{r}}$,

$C \rightarrow Q=\nabla \cdot W$

$D \rightarrow j_{\|}=\nabla \cdot \boldsymbol{J}$

The $\boldsymbol{J}_{\text {eq }}$-based method of characteristics has been applied in numerous studies, out of which we only show here one example, result from the analysis of an omega band passage over the MIRACLE network by Amm et al. (2005). The STARE radar measured the ionospheric electric field and the IMAGE magnetometer network the ground magnetic field (both being part of the MIRACLE network). In this case, simultaneous data from UV and X-ray measurements on the Polar satellites with the UVI and PIXIE instruments were used to estimate $\alpha$. Figure 7 (upper panel) shows that the technique can resolve in detail the eastward motion of the high Hall conductance areas associated with the bright auroral tongues of the omega-band. Inside the tongues, the analysis results into maximum Hall conductances of about $50-60^{\circ} \mathrm{S}$, while in the dark areas between the tongues very low conductances of just a few $\mathrm{S}$ prevail. While the results show a clear and consistent motion, they also show that the omega-bands are not just moving stationary structures, but significant temporal variation is found inside the moving forms. The associated FAC (Fig. 7, lower panel) show strong upward/downward FAC at the western/eastern flank of the tongues, respectively. In fact, most of the total westward ionospheric current is periodically carried to the ionosphere and away from it by these FAC. Thus, in the vicinity of the omega-bands, the substorm current wedge is not a continuous entity, but it is intermittently interrupted and consists of many smaller scale current wedges.

\section{1-dimensional methods}

The techniques presented in the previous chapters all operate in two horizontal dimensions. However, several types of situations exist in which data are only available along a single line, and not on a two-dimensional area. Among the most frequent of such situations are data from the pass of a single satellite, or from chains of magnetometers. In such cases, if the analysis of a single event is considered, two-dimensional analysis techniques naturally cannot be applied. Still, several of the 2-D techniques can be reduced to 1-dimensional variants (cf. Table 1) and thus be applied with data along a single line, provided that a horizontal direction exists in which the derivative of the measured data is, to a reasonable approximation, vanishing. This section presents 1-dimensional variants of several of the techniques that were reviewed in the previous section, and also discusses how to validate the 1-D assumption, and how to find an optimal coordinate system for the 1-D approach.

Before a 1-D analysis approach is applied to a data set, it is necessary to verify how well the 1-D assumption is realized in the case under study. While in theory, the term "1-D situation" is unambiguously defined as a situation with vanishing derivative of all measurements in one horizontal direction, in reality such a strict definition is hardly ever realized. For the practical application, the term "1-D situation" therefore refers to an approximation of the theoretical situation, which is defined by certain conditions that the measured data must fulfill. As examples, we discuss these conditions here for two typical cases in which 1-D methods are used: Magnetic field data from the overpass of a single low-orbiting satellite, and a from a magnetometer chain on the ground. Below, the 

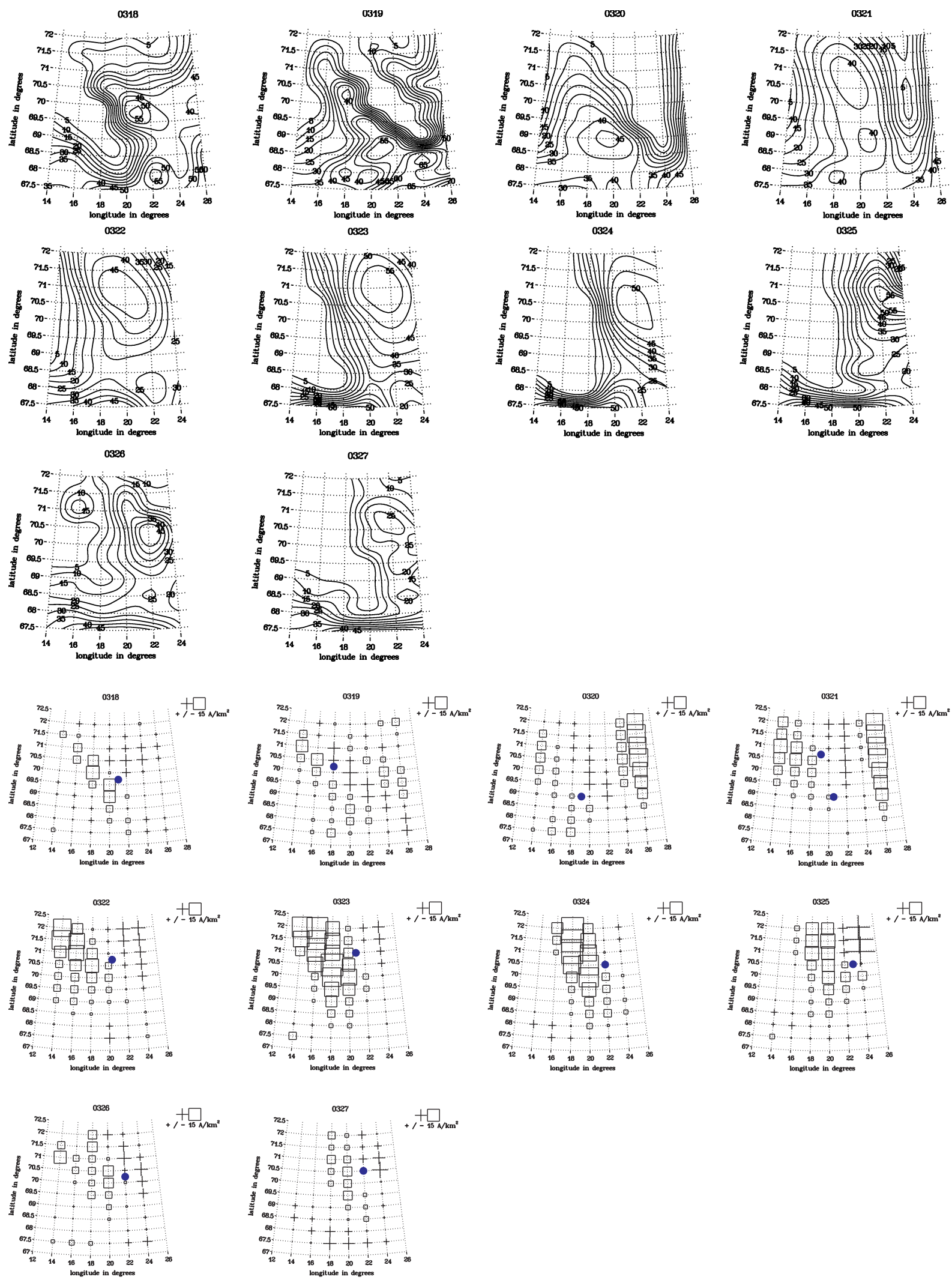

Fig. 7. Analysis results of the method of characteristics for an omega-band. Upper panel shows Hall conductance and lower panel fieldaligned currents $j_{\|}$(crosses denote downward, squares upward currents; dots give the positions of isolated conductance maxima above $69^{\circ}$ of latitude, cf. upper panel ). Reproduced from Fig. 6 of Amm et al. (2005) 
term "null gradient direction" corresponds to the direction in which the derivatives are (approximately) vanishing, and the term "1-D direction" to the direction perpendicular to this.

Using the assumption that the magnetic field disturbance that a low-orbiting satellite measures stems from the ionospheric currents and the FAC only, it is easy to see from an expansion of the current into 1-D SECS (Sect. 2.3) that the $B_{\mathrm{r}^{\prime}}$ and $B_{\theta^{\prime}}$ components of the magnetic field disturbance are not independent of each other for a 1-D situation, since both are solely generated by the $J_{\phi^{\prime}}$ current component. (Here the coordinate system $\left(r^{\prime}, \theta^{\prime}, \phi^{\prime}\right)$ refer to the spherical coordinates with respect to the pole of the 1-D SECS.) Juusola et al. (2007) have used this fact to apply the following procedure: For a given pole position of the 1-D SECS systems, the $J_{\phi^{\prime}}$ component of the current is computed only from the $B_{\mathrm{r}^{\prime}}$ component of the measured magnetic field disturbance. The resulting $J_{\phi^{\prime}}$ currents create a $B_{\theta^{\prime}}$,calc magnetic field disturbance, which in a perfect 1-D situation would be equal to the measured $B_{\theta^{\prime}}$. The magnetic data of an overpass is defined as 1-D if a position of the 1-D SECS pole can be found for which the error between $B_{\theta^{\prime}}$,calc and $B_{\theta^{\prime}}$ is smaller than a certain limit. The position of the 1-D SECS pole for which this error is smallest, which is found by an optimization technique, defines the optimum 1-D coordinate system for that particular overpass. Figure 8 illustrates the error in $B_{\theta^{\prime}}$ as a function of the location of the 1-D SECS pole position, and the path how the optimization technique finds the 1-D SECS pole location with the lowest $B_{\theta^{\prime}}$ error.

For the magnetic field disturbance data of a ground magnetometer chain, a similar approach is less suitable due to the more significant effect of magnetic field disturbances caused by currents induced into the Earth. Although it is possible to separate the internal and external contributions of the disturbance, the separation approach itself would already need the assumption of a 1-D situation (e.g. Untiedt and Baumjohann, 1993). Therefore, in order to check for a 1-D situation, in this case it is best to analyze data of some magnetometer stations which are aligned perpendicular to the main chain direction, if available. If the direction of the main chain is called $u$, and the horizontal direction perpendicular to it $v$, then a line of magnetometer stations in $v$ direction can be used to calculate the derivative of the magnetic field disturbance components in that direction. A good criterion for a 1-D situation is that the changing length $l_{Z}$ of each magnetic field component in $v$ direction is larger than the extent $l_{v}$ of the magnetometer line in $v$ direction, i.e.,

$l_{Z}=Z /(d Z / d v)>l_{v}$,

where $Z$ is used for any of the three magnetic field disturbance components. A similar procedure can easily be applied also to other data sets, like, e.g., ionospheric electric field data from a coherent scatter radar.
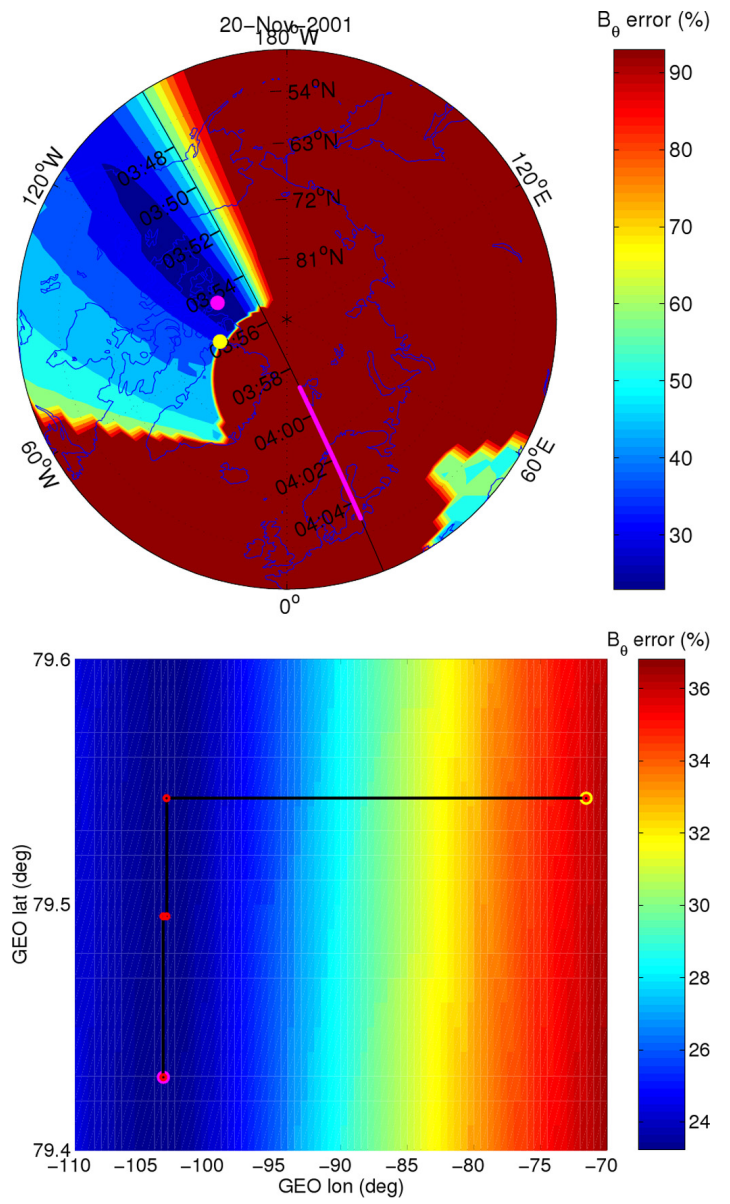

Fig. 8. Top: a typical example of the 1-D:ness optimization. The black line with the time stamps shows the track of the CHAMP satellite. The 1-D optimized part (overflight) is highlighted in magenta. The color coding displays the $B_{\theta^{\prime}}$ error for the overflight, as a function of the 1-D SECS pole location. The yellow dot shows the starting point for the optimization at the geomagnetic pole and the magenta dot the resulting 1-D SECS pole at $79^{\circ}$ latitude and $-103^{\circ}$ longitude with $B_{\theta^{\prime}}$ error $23 \%$. Bottom: a zoom in of the top panel showing also the steps taken during the optimization as red dots connected by black lines. Reproduced from Fig. 6 of Juusola et al. (2007).

\subsection{1-D ionospheric equivalent current determination}

Mersmann et al. (1979) used 1-D Fourier analysis (i.e. plane waves) to determine 1-D ionospheric equivalent currents from a chain of ground magnetometers. Olsen (1996) presented an analysis method where the ionospheric equivalent current sheet is presented as a sum of line currents, whose amplitudes are then fitted to the observed magnetic field. Although Olsen (1996) developed the method for the analysis of satellite observations, the same formalism may also be used with ground-based data. However, the 1-D Fourier analysis is hampered by the need to specify upper and lower limits to the resolved wavelengths, similar to the 2-D harmonic 
methods discussed in Sect. 3.1, and the line current method of Olsen (1996) does not properly take into account Earth's curvature in the direction perpendicular to the observatory chain.

To overcome these difficulties Vanhamäki et al. (2003) introduced a technique based on 1-D elementary systems. Except for the use of 1-D SECS instead of 2-D SECS, and the corresponding 1-D output of ionospheric equivalent currents along a single line, the technique is fully analogous to the 2-D variant presented in Sect. 3.3. Vanhamäki et al. (2003) have tested the technique using synthetic data sets with very good results, and then applied it with real data from the IMAGE and $210 \mathrm{MM}$ magnetometer chains (Fig. 1 and Yumoto, 2009, respectively).

\subsection{1-D method of characteristics}

As already shown by Inhester et al. (1992), in a 1-D case the integral Eqs. (44) and (45) for the method of characteristics reduce to the simple algebraic relation

$\Sigma_{\mathrm{H}}(u)=\frac{J_{\mathrm{eq}, \text { ion }, \mathrm{u}}(u)+\text { const }}{V_{u}(u)}$,

where $J_{\text {eq,ion, } u}$ is the ionospheric equivalent current in the 1$\mathrm{D}$ direction, as determined with the technique presented in Sect. 7.1. As Inhester et al. (1992) pointed out, if there is a location $u_{0}$ along the profile where $V_{u}=0$, then this sets the unknown constant in Eq. (51) to const $=-J_{\text {eq,ion, } u}\left(u_{0}\right)$. Moreover, the constant is also defined along the whole profile if at one single point of the profile a measurement of the Hall conductance exists. If such a measurement is not available, another suitable approach to estimate the constant is to assume a low background conductance in an area of the profile that is located outside of any electrojet activity, as determined by the ionospheric equivalent currents.

\subsection{1-D ionospheric currents and FAC determination from low-orbiting satellite magnetic data}

Magnetic data of a low-orbiting satellite can be used to determine both ionospheric currents and FAC if the situation can well be approximated as 1-D (see above), by expanding the magnetic disturbance recorded by the satellite into 1-D SECS. Similar to the techniques presented in Sects. 3.3 and 7.1 , the data is used to calculate optimum scaling factors of the 1-D SECS, which in turn determine both the ionospheric currents and FAC. This approach has first been presented by Juusola et al. (2006), and shown to be superior to previously used techniques to calculate ionospheric currents from satellite data (e.g. Olsen, 1996). One major advantage of using 1-D SECS for this type of analysis is that by placing SECS poles at certain distances, a spatial scale is selected naturally, which is the same for both components of the resulting ionospheric currents and for the FAC. Therefore, it makes sense to combine the results for the different current components

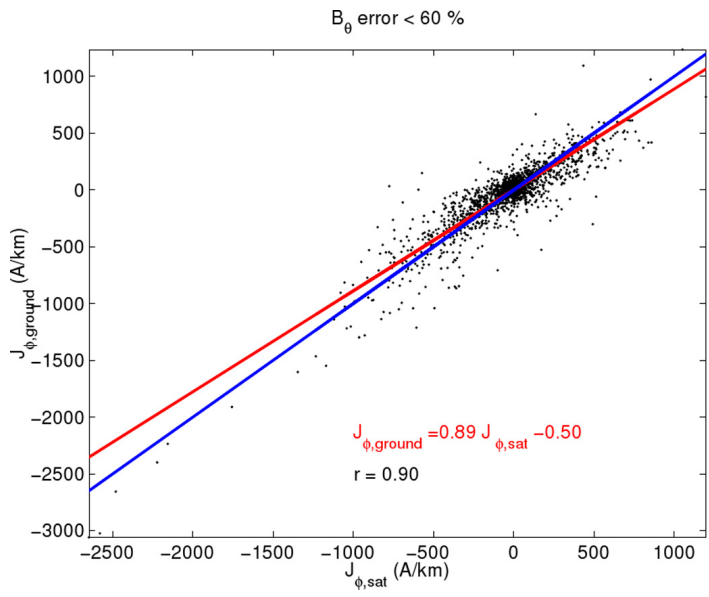

Fig. 9. Scatter plot of $J_{\phi}$ determined from ground-based measurements (IMAGE) by the 2-D SECS method versus $J_{\phi}$ determined from satellite-based measurements (CHAMP) by the 1S SECS method. The ground-based $J_{\phi}$ is averaged over the approximately $4 \mathrm{~min}$ it takes for the satellite to pass over IMAGE. In red is shown a line fitted to the points, and the linear correlation coefficient is denoted by $r$. A line passing through the origin with a unit slope is drawn in blue. To create this plot, data from 124 satellite passes over IMAGE with an error smaller than $60 \%$ during 2001 and 2002 were used. Reproduced from Fig. 5 of Juusola et al. (2007).

for further analysis, even though the magnetic field caused by the FAC is local to the satellite while the one caused by the ionospheric currents is remote. It should be noted that the 1-D SECS technique also makes it possible to analyze low-orbiting satellite and ground-based magnetometer data simultaneously, by combining the techniques of Sect. 7.1 and of this section into a single 1-D SECS expansion.

This technique has been extensively used with data of the CHAMP satellite (e.g. Ritter et al., 2004), which passes over the auroral ionosphere at about $400 \mathrm{~km}$ altitude. Juusola et al. (2007) have analyzed more than 6000 passes between $55^{\circ}$ and $76.5^{\circ}$ northern geomagnetic latitude during 2001 and 2002 which satisfy their 1-D condition. In order to crosscheck the resulting ionospheric currents with results from ground-based magnetometer analysis, the authors selected passes of CHAMP over the MIRACLE network (Fig. 1), and used the technique presented in Sect. 3.3 to determine the 2-D ionospheric equivalent currents from the MIRACLE data. They then compared 1-D profiles of these results along each CHAMP overpass with the results of ionospheric currents from the 1-D SECS analysis of the CHAMP data. As can be seen from Fig. 9, the correspondence between the two is very good with a correlation coefficient of 0.9 , especially when taking into account that the ground-based technique provides equivalent currents and the satellite-based technique real ones. In practice, the results of Juusola et al. (2007) show that the ground-based and satellite results are interchangeable when the satellite passes over a ground magnetometer array. 

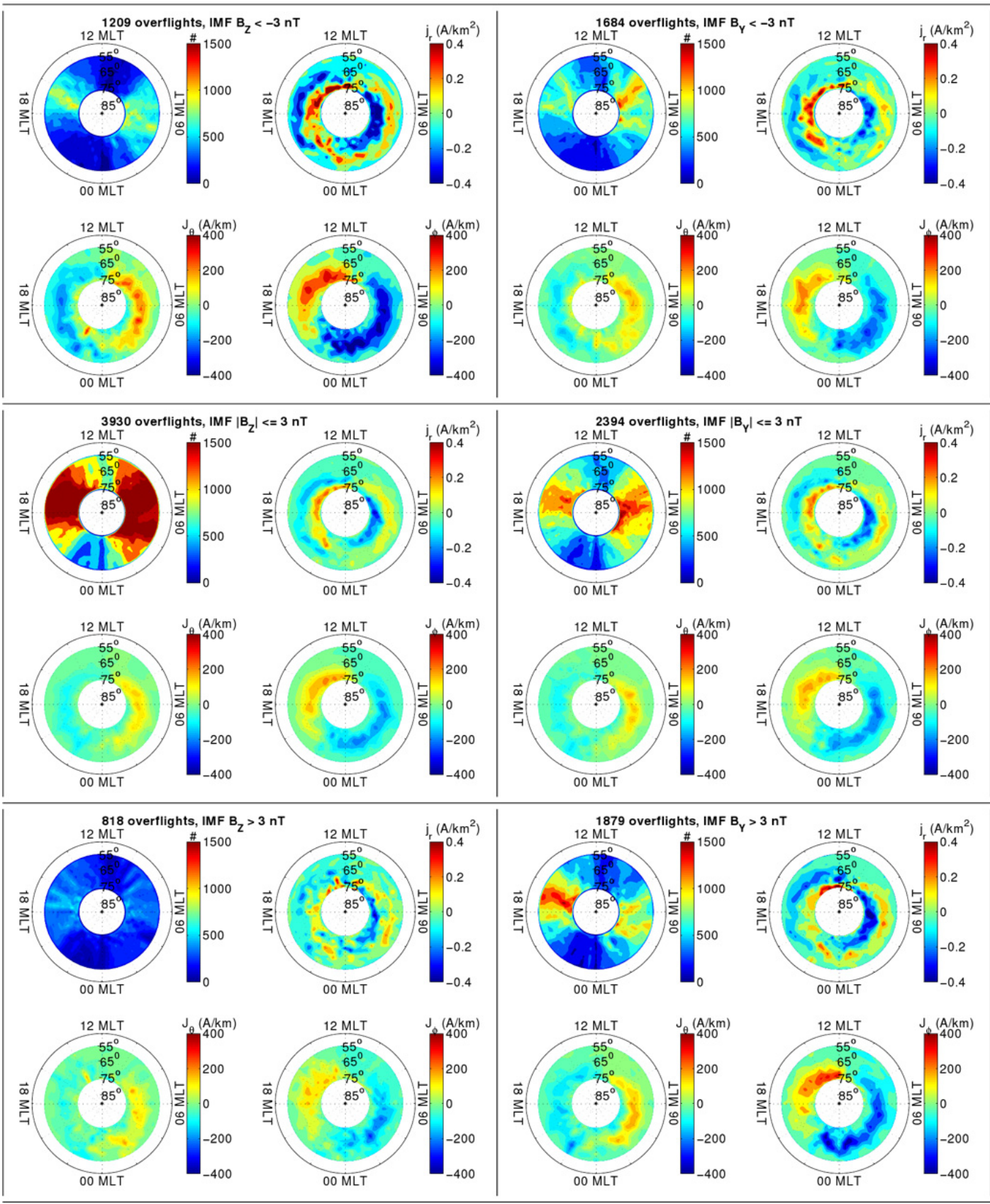

Fig. 10. Current components $J_{\mathrm{r}}$ (FAC), $J_{\theta}$ and $J_{\phi}$ as function of magnetic latitude and MLT, binned with respect to the IMF BZ and BY. There are bins for negative $(<3 \mathrm{nT})$, around zero $(<|3| \mathrm{nT})$ and positive $(>3 \mathrm{nT})$ values of both components. The bin and the number of overpasses used to construct the four plots are denoted on top of each set. Resolution of the plots is $0.5 \mathrm{~h}$ in MLT and $1^{\circ}$ in latitude. Top left plot of each panel shows the distribution of CHAMP measurements in the data set. Reproduced from Fig. 12 of Juusola et al. (2007).

Juusola et al. (2007) combined the results of all CHAMP passes into a statistical study of the dependence of ionospheric currents and FAC of the general geomagnetic activity level, of season, and of the interplanetary magnetic field (IMF). As an example, Fig. 10 shows the dependence of $J_{\mathrm{r}}$ (FAC), $J_{\theta}$ and $J_{\phi}$ of the IMF, in addition to the data coverage for each IMF bin, as a function of geomagnetic latitude and MLT. Further, in a 1-D case it is possible to calculate the value of the Hall-to-Pedersen conductance ratio $\alpha$ from the magnetic data alone, simply as

$\alpha=-J_{\phi} / J_{\theta}$.

The parameter $\alpha$ is an important factor in ionospheremagnetosphere physics, as it is representative for the mean 


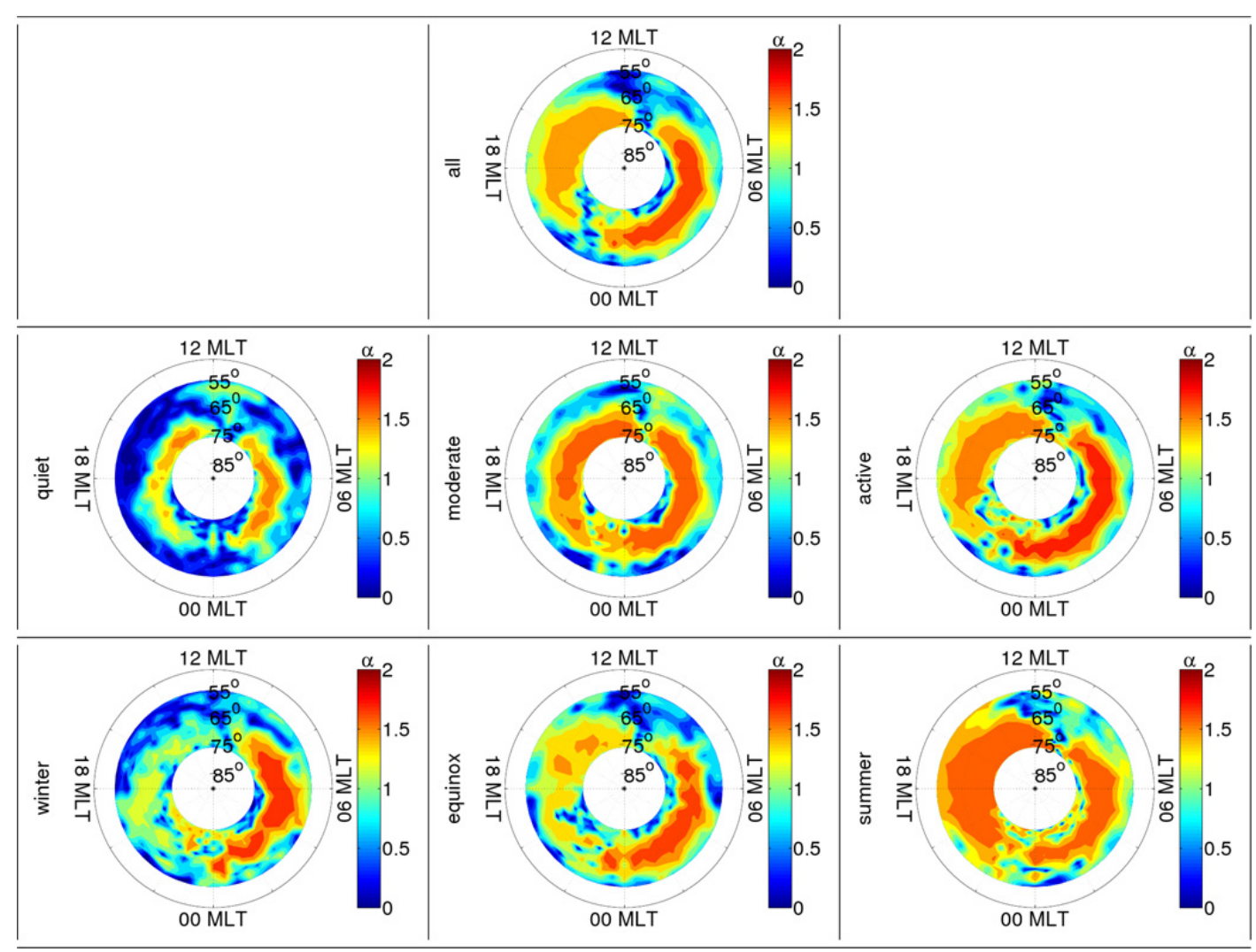

Fig. 11. Average $\alpha$ during 2001-2002 as a function of magnetic latitude and MLT. The resolution of the plots is $1^{\circ}$ in latitude and $0.5 \mathrm{~h}$ in MLT, and $\alpha$ is given according to the color bar on the right hand side of each plot. The upmost plot is constructed using all available data, the plots in the middle row are binned with respect to activity and those in the bottom row with respect to season (the bin is denoted on the left hand side of each plot). Reproduced from Fig. 15 of Juusola et al. (2007).

energy of precipitating particles (Robinson et al., 1987), and is needed as input for other techniques, such as the method of characteristics (Sect. 6). Juusola et al. (2007) presented analytical, average relations between $\alpha$ and $J_{\phi}$, separately for westward and eastward electrojet conditions, and for summer, equinox and winter. They also presented the results in global, statistical maps of $\alpha$, reproduced in Fig. 11, which constitutes the most extensive data-based description of this parameter over the whole auroral zone as yet. Recently, using the same data set and technique, Juusola et al. (2009) also analyzed the dependence of ionospheric currents and FAC on the solar wind dynamic pressure.

\section{Induction in ionospheric electrodynamics}

It is usually assumed that inductive phenomena in the ionosphere are negligible. This is a central assumption in many of the analysis methods reviewed in Table 1, because it allows us to present the ionospheric electric field in terms of a potential, as in Eq. (30). However, in certain very dynamical situations this assumption is not valid, and the electric field may have a considerable induced rotational part.
Those methods where the electric field is an input parameter (e.g. the method of characteristics) can in principle handle inductive electric fields. However, in practice the input $\boldsymbol{E}$ field is often preprocessed in such a way that the inductive rotational part of the measured field may be lost in the process. For example, the SuperDARN potential mapping technique (Ruohoniemi and Baker, 1998) is based on the assumption $\nabla \times \boldsymbol{E}=0$, although recently Amm et al. (2010) presented a technique that allows to pertain the curl when $\boldsymbol{E}$ is calculated from line-of-sight radar data.

Ionospheric self-induction has been studied in the framework of Alfvén wave reflection at the ionospheric boundary of a magnetic flux-tube. When an incident shear Alfvén wave carrying a potential electric field is reflected from the non-isotropically conducting ionosphere, the reflected wave consists of both shear and fast mode waves. This induction induced mode coupling has been noted for some time (e.g. Allan and Knox, 1979a,b), although many authors have considered it negligible (e.g. Glassmeier, 1984). Yoshikawa and Itonaga (1996) studied the reflection and mode conversion coefficients in detail, and concluded that ionospheric self-induction plays a significant role at large scale-lengths 


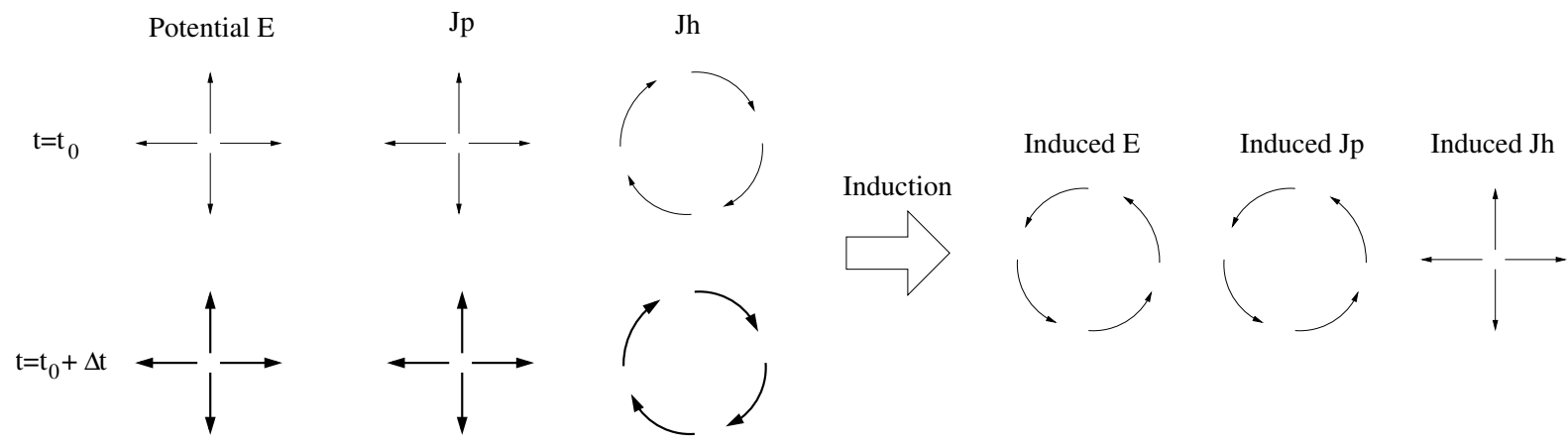

Fig. 12. Lenz's law in ionospheric electrodynamics (assuming uniform conductances and downward pointing background magnetic field). Changes in the potential electric field and associated currents $\left(\boldsymbol{J}_{\mathrm{P}}\right.$ and $\left.\boldsymbol{J}_{\mathrm{H}}\right)$ create rotational induced electric field. Direction of induced $\boldsymbol{E}$ is such that induced Pedersen currents oppose the change in rotational currents. This means that the induced Hall currents enhance the change in divergent currents and associated FAC. In the case of non-uniform ionospheric conductances the situation is more complicated.

and/or when Hall conductance is large. Later studies by e.g. Buchert (1998), Yoshikawa and Itonaga (2000), Lysak and Song (2001), Lysak (2004) and Sciffer et al. (2004), have confirmed these results and investigated further the reflection process and the propagation of the shear and fast mode waves in the ionosphere.

While the Alfvén wave models have given us a better understanding of the ionospheric inductive phenomena, geomagnetic pulsations and coupling of the ionosphere and magnetosphere, they are not really suitable for event studies. The main problem is that in all these models the spatial and temporal distribution of the incident Alfvén waves above the ionosphere is assumed to be known. This is quite a restrictive assumption in practical studies, because the incident wave pattern is very hard to measure. To the authors' knowledge there seems to be no empirical models of Alfvén wave patterns related to some specific ionospheric events. In principle one could use a magnetospheric MHD simulation as an input in the Alfvén wave scheme. However, current simulations use electrostatic ionospheric solvers and it would not be straightforward to couple them to an ionospheric Alfvén wave solver (Janhunen, 1998). Only very recently, Yoshikawa et al. (2010) presented a plausible coupling scheme for this purpose.

\subsection{Lenz's law in the ionosphere}

Lenz's law states that the direction of the induced electric field in a loop of wire is such that the induced current opposes the change of magnetic flux through the loop. At first one might think that according to Lenz's law the induced ionospheric currents should oppose the original currents. However, this is not always the case, as can be seen in the examples presented by Vanhamäki et al. (2007). In many cases induction seems to enhance the change in FAC flowing between the ionosphere and magnetosphere. This somewhat counterintuitive result is explained in Fig. 12, which is a schematic presentation of Lenz's law in ionospheric electro- dynamics. It should be noted that only rotational currents are associated with the radial component of magnetic field that goes through the ionospheric current layer. The induced currents oppose the change in the rotational current, and hence also the change of magnetic flux through the ionospheric plane, but enhance the change in the divergent currents. This tendency of inductive currents to enhance the change of FAC was also noted by Buchert (1998) and Yoshikawa and Itonaga (2000).

\subsection{Including inductive effects in existing data-analysis methods}

It is possible to modify the existing data-analysis methods so that inductive effects are included in the analysis selfconsistently. One obvious requirement is that the analysis is performed on time series, rather than individual time steps, since induction is related to the time derivative of the magnetic field.

Recently, Vanhamäki (2010) developed an inductive ionospheric solver for MHD simulations. The same formalism can also be used for the KRM method, as discussed in Sect. 5.3, where the electrostatic solution given by Eq. (39) was derived. The electrodynamic (inductive) solution is obtained by relating the curl of the electric field, curle, to the divergence- and curl-free current systems, $\mathfrak{I}_{\mathrm{CF}}$ and $\mathfrak{I}_{\mathrm{DF}}$, respectively, via Faraday's and Ampere's laws.

Using the same notation as in in Sect. 5.3, Faraday's law can be written in terms of SECS as

$\mathfrak{c u r l e}=-\frac{\partial \mathfrak{B}_{\mathrm{r}}}{\partial t}$,

where the vector $\mathfrak{B}_{\mathrm{r}}$ contains the radial magnetic field at the grid points $\boldsymbol{r}_{u}$,

$\mathfrak{B}_{\mathrm{r}}=\left[\begin{array}{llll}B_{\mathrm{r}}\left(\boldsymbol{r}_{1}\right) & B_{\mathrm{r}}\left(\boldsymbol{r}_{2}\right) & B_{\mathrm{r}}\left(\boldsymbol{r}_{3}\right) \ldots\end{array}\right]^{T}$.

The vector $\mathfrak{B}_{\mathrm{r}}$ can be written as a function of the current as

$\mathfrak{B}_{\mathrm{r}}=\mathbf{N}_{1} \cdot \mathfrak{I}_{\mathrm{CF}}+\mathbf{N}_{2} \cdot \mathfrak{I}_{\mathrm{DF}}$. 
The matrices $\mathbf{N}_{1,2}$ can be obtained using the expressions for the magnetic fields of individual elementary systems, as outlined by Vanhamäki (2010). In the case of a vertical background magnetic field $\mathbf{N}_{1}=0$.

Equations (39), (53) and (55) can be combined as

$\mathbf{L}_{1} \cdot \mathfrak{I}_{\mathrm{CF}}+\mathbf{L}_{2} \cdot \mathfrak{I}_{\mathrm{DF}}=-\frac{\partial}{\partial t}\left(\mathbf{N}_{1} \cdot \mathfrak{I}_{\mathrm{CF}}+\mathbf{N}_{2} \cdot \mathfrak{I}_{\mathrm{DF}}\right)$.

Similar to the electrostatic solution in Eq. (39), also this equation can be integrated step-by-step in time with either $\mathfrak{I}_{\mathrm{DF}}$ (equivalent current) or $\mathfrak{I}_{\mathrm{CF}}$ (FAC) as the input data, resulting in an inductive KRM method or FAC-based solver, respectively.

Also the analysis method developed by Takeda (2008) takes magnetospheric FAC distributions and ionospheric conductances as input data, but the mathematical approach is somewhat different from Vanhamäki (2010). Takeda (2008) represent the divergence-free current with a potential $\psi_{J}$, as in Eq. (6), while the curl-free part of the current is expanded as a sum of simple vector systems equivalent to the CF SECS used by Vanhamäki (2010). On a global scale, with FAC as input data, the two formulations should be equivalent. However, on mesoscales the boundary conditions are somewhat different, and Takeda (2008) did not consider using ground magnetic field as input, instead of FAC.

\subsection{Examples of ionospheric self-induction}

Vanhamäki et al. (2006) presented a calculation method that solves the ionospheric induction problem self-consistently using the ionospheric potential electric field and conductances as input. This calculation method can handle nonuniform, time-dependent ionospheric conductances and electric fields of any geometry. Vanhamäki et al. (2007) applied this method to several realistic, data-based models of typical ionospheric current systems, including an intensifying electrojet, a westward traveling surge (WTS) and an $\Omega$-band. In the WTS and $\Omega$-band models the induced electric field is concentrated in a small area where the time derivatives are largest, as demonstrated in Fig. 13. In the electrojet model the induced field is significant over a large part of the jet area. In these examples the induced electric field has typical values of a few $\mathrm{mV} \mathrm{m}^{-1}$, which nevertheless amounts to several tens of percents of the potential electric field present at the same locations. The induced electric field is associated with horizontal and field-aligned currents, that modify the overall structure of the current systems. Especially the induced FAC are often comparable to the non-inductive FAC, and may thus significantly modify the coupling between the ionosphere and magnetosphere in the most dynamical situations.

Takeda (2008) found that global current systems with a period of less than 4 min are significantly affected by the induction field. The model results are consistent with the observed characteristics of the preliminary impulse of storm commencement.
Vanhamäki (2010) demonstrated the SECS-based inductive ionospheric solver, by analyzing the same WTS and $\Omega$ bands models as studied by Vanhamäki et al. (2007) and Vanhamäki and Amm (2007) with earlier versions of the algorithm (discussed in Sects. 8.2 and 5.3, respectively). Figure 14 illustrates the difference between the electrostatic (Eq. 39) and electrodynamic (Eq. 56) solutions for the WTS. The temporal evolution was created by moving the static model at $10 \mathrm{~km} \mathrm{~s}^{-1}$ westward.

Even though the induced rotational part of the electric field is rather small, only $\sim 0.8 \mathrm{~V} \mathrm{~km}^{-1}$ in this case, the difference between the static and electrodynamic solutions in Fig. 14 is significant. The rotational electric field is concentrated in the highly conducting "head" of the WTS, where also the static potential electric field is suppressed. Consequently, the induced field aligned currents contribute about $20-30 \%$ of the total FAC in this area. As the total FAC is a fixed input parameter in this analysis method, also the potential part of $\boldsymbol{E}$ is indirectly modified by induction. This effect does not appear in the results obtained by Vanhamäki et al. (2007), see Fig. 13, as in their calculation method the potential electric field is a fixed input parameter.

\section{New development}

In this section we discuss some selected new developments related to the data-analysis tools reviewed above. In Sect. 9.1 we outline two possible extensions of the method of characteristics that may have some applications with currently available data. Future multi-satellite missions at low Earth orbit enable a more extensive determination of ionospheric currents and FAC than possible with the 1-D methods discussed in Sect. 7.3. These new possibilities are explored in Sect. 9.2. We conclude this outlook by briefly discussion the possibilities for 3-D ionospheric data-analysis in Sect. 9.3.

\subsection{New versions of the method of characteristics}

In the presently used formulations of the method of characteristics discussed in Sect. 6, it was assumed that the conductance ratio $\alpha$ is a known input parameter. However, from a technical point of view it is equally possible to use either the Hall or Pedersen conductance as an input parameter, instead of $\alpha$. If $\Sigma_{\mathrm{P}}$ is known, we can start with relation

$\left[\nabla \times\left(\Sigma_{\mathrm{H}} \boldsymbol{E}\right)\right]_{\mathrm{r}}=\left[\nabla \times\left(\boldsymbol{J}_{\text {eq,ion }}-\Sigma_{\mathrm{P}} \boldsymbol{E}\right)\right]_{\mathrm{r}}$,

where the right side is known from the input data. This can be written out as

$\nabla \Sigma_{\mathrm{H}} \cdot\left(\boldsymbol{E} \times \hat{\boldsymbol{e}}_{\mathrm{r}}\right)+\Sigma_{\mathrm{H}} \nabla \cdot\left(\boldsymbol{E} \times \hat{\boldsymbol{e}}_{\mathrm{r}}\right)=\left[\nabla \times\left(\boldsymbol{J}_{\text {eq,ion }}-\Sigma_{\mathrm{P}} \boldsymbol{E}\right)\right]_{\mathrm{r}},(57)$

which is completely analogous to Eq. (43). This thus far overlooked approach may have some applications, as Senior et al. (2008) showed that $\Sigma_{\mathrm{P}}$ can be estimated quite accurately from all-sky camera images taken at $557.7 \mathrm{~nm}$. 

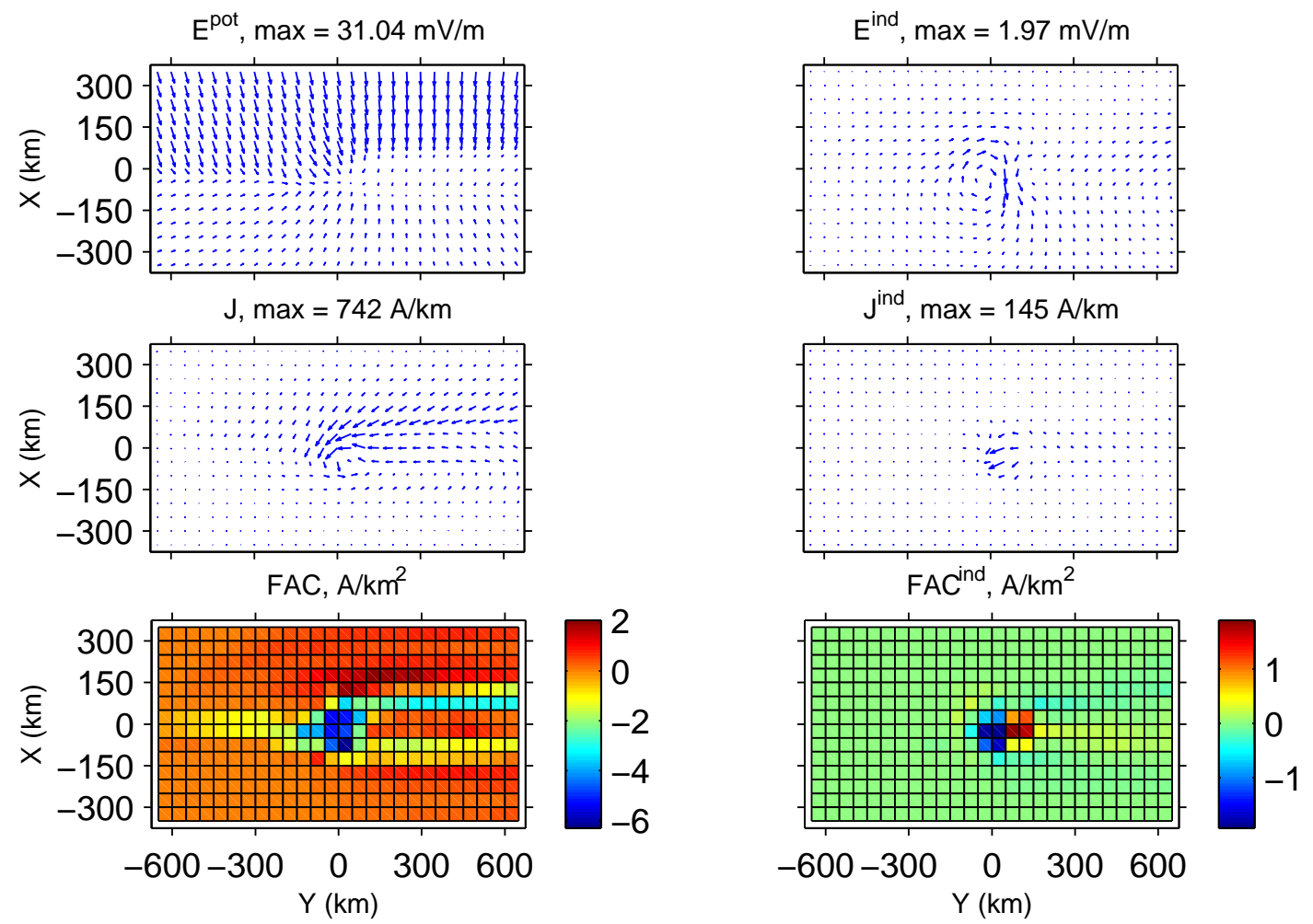

Fig. 13. Lefthand panels show the potential electric field and associated horizontal and field-aligned currents of the quasi-static westward traveling surge model. Righthand panels show the induced electric field and associated currents that are created when the WTS system is moving at $10 \mathrm{~km} \mathrm{~s}^{-1}$ westward. Modified from Fig. 8 of Vanhamäki et al. (2007).

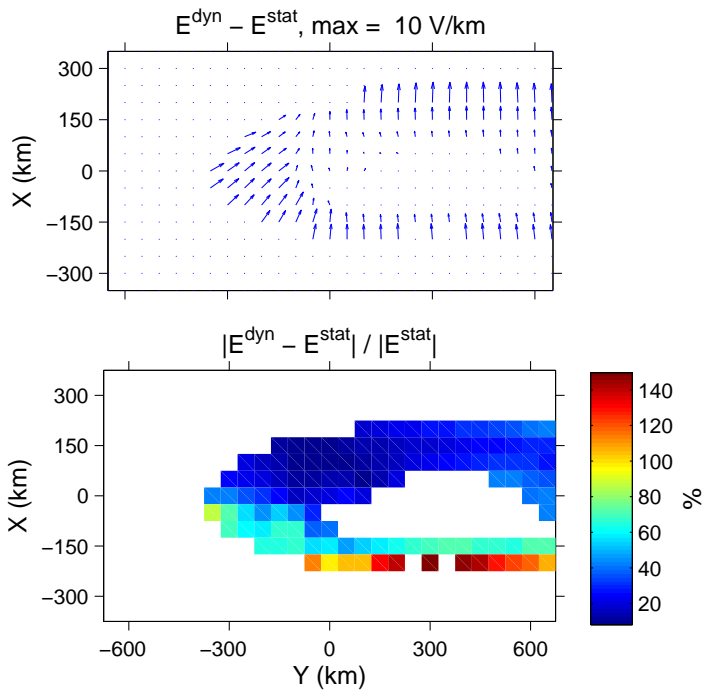

Fig. 14. Results for the WTS model. Upper panel shows the difference between the electric fields obtained from static $\left(\boldsymbol{E}^{\text {stat }}\right)$ and electrodynamic $\left(\boldsymbol{E}^{\text {dyn }}\right)$ solvers. The lower panel shows the percentage difference in electric field magnitude. Only those areas where $\left|\boldsymbol{E}^{\text {stat }}\right|>5 \mathrm{~V} \mathrm{~km}^{-1}$ and $\Sigma_{\mathrm{H}}>5 \mathrm{~S}$ are shown. Reproduced from Vanhamäki (2010).
Another extension of the present analysis methods was mentioned in Sect. 4. If we obtain the total current $\boldsymbol{J}$ from FAC and ground magnetic field, and further estimate $\alpha$ (e.g. from the current itself, see Juusola et al., 2007), we can formulate a new " $\boldsymbol{J}$-based" method of characteristics. The central equation is obtained from the curl of inverted Ohm's law, $\left(\nabla \times\left[\left(\Sigma_{\mathrm{P}} \boldsymbol{J}+\Sigma_{\mathrm{H}} \hat{\boldsymbol{e}}_{\mathrm{r}} \times \boldsymbol{J}\right) /\left(\Sigma_{\mathrm{P}}^{2}+\Sigma_{\mathrm{H}}^{2}\right)\right]\right)_{\mathrm{r}}=(\nabla \times \boldsymbol{E})_{\mathrm{r}}$.

This can be written as

$\nabla R_{\mathrm{H}} \cdot\left(\boldsymbol{J} / \alpha \times \hat{\boldsymbol{e}}_{\mathrm{r}}+\boldsymbol{J}\right)+R_{\mathrm{H}} \nabla \cdot\left(\boldsymbol{J} / \alpha \times \hat{\boldsymbol{e}}_{\mathrm{r}}+\boldsymbol{J}\right)=(\nabla \times \boldsymbol{E})_{\mathrm{r}},(58)$

where $R_{\mathrm{H}}=\Sigma_{\mathrm{H}} /\left(\Sigma_{\mathrm{P}}^{2}+\Sigma_{\mathrm{H}}^{2}\right)$. Also this equation has the same form as Eq. (43) and can be solved in a similar way for $R_{\mathrm{H}}$. The curl of $\boldsymbol{E}$ may either be approximated as zero, or calculated from the known current system.

However, these variants of the method of characteristics have not yet been thoroughly tested nor applied with real data.

\subsection{Analysis of multi-satellite magnetic data}

Future multi-satellite mission at low Earth orbit give some information about the cross-track gradients, so the 1-D analysis methods discussed in Sect. 7.3 must be generalized in order to get full benefit from the available measurements. 
For example, in the planned Swarm mission up to 3 close by satellites tracks are available. Ritter and Lühr (2006) applied the curl-B technique to simulated Swarm measurements and were able to derive the ionospheric FAC uniquely in the general case without any assumptions on vanishing gradients to some specific direction. This result is not possible to obtain with single-satellite techniques.

In terms of the application of the SECS technique to derive ionospheric currents (as it was presented for a single satellite analysis in Sect. 7.3), in the case of multiple satellites it is possible to extend the analysis area from a single line to an extended strip that contains the footprint trajectories of all satellites used. Within this strip, using a combination of 1-D and 2-D SECS as basis functions, the total ionospheric currents and FAC can be obtained from the magnetic field data provided by the satellites. The exact details and what assumptions are needed for this procedure depend on the number of satellites available, and need to be tested in future work.

\subsection{3-D modeling and data-analysis}

All the methods listed in Table 1 use one common approximation that is not explicitly mentioned in the table: They all assume a thin sheet ionosphere, where all vertical structure is integrated into an infinitely thin layer. This approximation originally stems from the analysis of ground-based magnetometers that alone, due to basic physical reasons (Sect. 2.2), can only measure altitude integrated effects. In present day science, it is partly used in order to simplify the analysis, but also because lack of 3-dimensional input data. However, new radar systems such as AMISR (Nicolls and Heinselman, 2007) can already now provide some 3-D measurements, and the amount of available data will increase in the near future with upcoming missions like the Swarm ionospheric multisatellite project, or the EISCAT 3-D radar system. Consequently, also data-analysis methods have to be formulated in 3-D, so that the new observations can be fully utilized. This line of future development has been discussed by Amm et al. (2008) in a recent review article.

\section{Summary}

We have reviewed selected data-analysis methods suitable to be used with data from a mesoscale ionospheric observation network (some hundred or a few thousand $\mathrm{km}$ across), such as MIRACLE illustrated in Fig. 1, possibly in combination with satellite measurements. Table 1 gives an overview of the reviewed methods, including input and output data as well as assumption used in the method (if any).

As discussed in Sect. 2, we need 3 separate (scalar) input parameters in order to solve the full electrodynamic state of the ionosphere. In some cases a smaller number may be sufficient for a partial solution, like in the elementary sys- tem method, where ground magnetic field and FAC are used to determine the horizontal current system, but the electric field remains unknown unless conductance data are available. Looking at Table 1, and including the extensions of the method of characteristics as discussed in Sect. 9.1, it would appear that an analysis method has been developed for most, if not all of those input data sets that allow for a full solution. However, there may be several undeveloped methods that give a partial, but still useful solution of ionospheric electrodynamics.

Several methods listed in Table 1 use ground magnetic measurements as the input data. Typically $\boldsymbol{B}_{\mathrm{G}}$ is first converted to ionospheric equivalent current, as discussed in Sect. 3. In principle the ground magnetic disturbance should be separated into internal and external parts, but in practice performing the separation with a too sparse magnetometer array may cause larger errors than simply neglecting the often small and fairly uniform internal part (Pulkkinen et al., 2003b).

The KRM and FAC-based ionospheric solvers were discussed in Sect. 5. Traditionally these methods have been used only in global studies, because the unknown boundary conditions may affect the solution considerably. The AMIE/KRM combination and the SECS-based formulation of KRM discussed in Sects. 5.2 and 5.3 are more suitable for mesoscale analysis, although the boundary conditions still have some effect the solution, especially in the FAC-based method introduced by Vanhamäki (2010). Also the availability of conductance data is a limiting factor, especially in mesoscale event studies, where high-resolution Hall and Pedersen conductances are required as the input data.

In the method of characteristics, discussed in Sect. 6, we can use the conductance ratio $\alpha=\Sigma_{\mathrm{H}} / \Sigma_{\mathrm{P}}$ as an input parameter. The ratio is easier to estimate than the conductances themselves, but the downside is that the electric field and either FAC or equivalent current are required as input. However, the method of characteristics is well suited for mesoscale analysis, as it provides explicit estimate on how much the solution is affected by the choice of boundary conditions, and in some cases boundary condition are not required at all. In Sect. 9.1 we briefly discussed some possible extensions of the method of characteristics.

The 1-D methods discussed separately in Sect. 7 are extremely useful when analyzing data from meridional magnetometer chains, single satellite passes or similar intrinsically 1-dimensional situations. Most of the methods listed in Table 1 have both 1-D and 2-D versions. A notable exception is the last one, 1-D SECS method for analyzing satellite-based magnetic measurements, for which oly a 1-D variant exists as yet. It may be possible to generalize this method so that some 2-D structures can be deduced from multiple simultaneous satellite passes, as discussed in Sect. 9.2.

In Sect. 8 we discuss analysis techniques where ionospheric induction is taken into account. Inductive effects are often neglected, but induction is known to modify the 
ionospheric reflection of Alfvén waves and it may also play a non-negligible role in the most dynamic phenomena, such as substorms. Faraday's law can be included in the KRM method and FAC-based ionospheric solver, as discussed in Sect. 8.2, so the assumption $(\nabla \times \boldsymbol{E})_{\mathrm{r}}=0$ as listed in Table 1 is not absolutely necessary with these methods, although it simplifies the analysis.

We expect that in the future the importance of 3-D ionospheric modeling will increase, with new instruments and tomographic techniques coming to use. Some phenomena, such as vertical current closure inside the ionosphere and altitude dependent contribution of polarization space charges to the electric field, both of which may be important e.g. in a Cowling channel, can be handled correctly only in 3-D ionosphere (Amm et al., 2008). Also inductive phenomena are modified in a realistic 3-D ionosphere, due to vertical current loops between the Hall and Pedersen currents.

Acknowledgements. The work of $\mathrm{H}$. Vanhamäki is supported by the Academy of Finland (project number 126552).

Editor-in-Chief M. Pinnock thanks two anonymous referees for their help in evaluating this paper.

\section{References}

Ahn, B.-H., Kamide, Y., Kroehl, H. W., Candidi, M., and Murphree, J. S.: Substorm changes of the electrodynamic quantities in the polar ionosphere: CDAW 9, J. Geophys. Res., 100, 2384523856, 1995

Ahn, B.-H., Richmond, A. D., Kamide, Y., Kroehl, H. W., Emery, B. A., de la Beaujardiére, O., and Akasofu, S. I.: An ionospheric conductance model based on ground magnetic disturbance data, J. Geophys. Res., 103, 14769-14780, 1998.

Aksnes, A., Amm, O., Stadsnes, J., Østgaard, N., Germany, G. A., Vondrak, R. R., and Sillanpää, I.: Ionospheric conductances derived from satellite measurements of auroral UV and X-ray emissions, and ground-based electromagnetic data: a comparison, Ann. Geophys., 23, 343-358, doi:10.5194/angeo-23-343-2005, 2005.

Allan, W. and Knox, F. B.: A dipole field model for axisymmetric Alfvén waves with finite ionosphere conductivities, Planet. Space Sci., 27, 79-85, 1979a.

Allan, W. and Knox, F. B.: The effect of finite ionosphere conductivities on axisymmetric toroidal Alfvén wave resonances, Planet. Space Sci., 27, 939-950, 1979b.

Amm, O.: Direct determination of the local ionospheric Hall conductance distribution from two-dimensional electric and magnetic field data: Application of the method using models of typical ionospheric electrodynamic situations, J. Geophys. Res., 100, 21473-21488, 1995.

Amm, O.: Ionospheric elementary current systems in spherical coordinates and their application, J. Geomagnetism Geoelectricity, 49, 947-955, 1997.

Amm, O.: Method of characteristics in spherical geometry applied to a Harang-discontinuity situation, Ann. Geophys., 16, 413424, doi:10.1007/s00585-998-0413-2, 1998.
Amm, O.: The elementary current method for calculating ionospheric current systems from multisatellite and ground magnetometer data, J. Geophys. Res., 106, 24843-24855, 2001.

Amm, O.: The method of characteristics for calculating ionospheric electrodynamics from multi-satellite and groundbased radar data, J. Geophys. Res., 107(A10), 1270, doi:10.1029/2001JA005077, 2002.

Amm, O. and Viljanen, A.: Ionospheric disturbance magnetic field continuation from the ground to the ionosphere using spherical elementary current systems, Earth Planets Space, 51, 431-440, 1999.

Amm, O., Engebretson, M., Hughes, T., Newitt, L., Viljanen, A., and Watermann, J.: A traveling convection vortex event study: Instantaneous ionospheric equivalent currents, estimation of field-aligned currents, and the role of induced currents, J. Geophys. Res., 107(A11), 1334, doi:10.1029/2002JA009472, 2002.

Amm, O., Aksnes, A., Stadsnes, J., stgaard, N., Vondrak, R. R., Germany, G. A., Lu, G., and Viljanen, A.: Mesoscale ionospheric electrodynamics of omega bands determined from ground-based electromagnetic and satellite optical observations, Ann. Geophys., 23, 325-342, doi:10.5194/angeo-23-325-2005, 2005.

Amm, O., Aruliah, A., Buchert, S. C., Fujii, R., Gjerloev, J. W., Ieda, A., Matsuo, T., Stolle, C., Vanhamki, H., and Yoshikawa, A.: Towards understanding the electrodynamics of the 3-dimensional high-latitude ionosphere: present and future, Ann. Geophys., 26, 3913-3932, doi:10.5194/angeo-26-39132008, 2008.

Amm, O., Grocott, A., Lester, M., and Yeoman, T. K.: Local determination of ionospheric plasma convection from coherent scatter radar data using the SECS technique, J. Geophys. Res., 115, A03304, doi:10.1029/2009JA014832, 2010.

Anderson, B. J., Takahashi, K., and Toth, B. A.: Sensing global Birkeland currents with Iridium engineering magnetometer data, Geophys. Res. Lett., 27(24), 4045-4048, 2000.

Brekke, A.: Physics of the upper polar atmosphere, John Wiley \& Sons, ISBN 0-471-96018-7, 1997.

Buchert, S.: Magneto-optical Kerr effect for a dissipative plasma, J. Plasma Phys., 59, 39-55, 1998.

Chapman, S. and Bartels, J.: Geomagnetism, vol. II, Oxford University Press, New York, 1940.

Escoubet, C. P., Fehringer, M., and Goldstein, M.: Introduction: The Cluster mission, Ann. Geophys., 19, 1197-1200, doi:10.5194/angeo-19-1197-2001, 2001.

Fukushima, N.: Generalized theorem for no ground magnetic effect of vertical currents connected with Pedersen currents in the uniform-conductivity ionosphere, Rep. Ionos. Space. Res. Japan, 30, 35-40, 1976.

Fuller-Rowell, T. J. and Evans, D. S.: Height-integrated Pedersen and Hall conductivity patterns inferred from the TIROS-NOAA satellite data, J. Geophys. Res., 92, 7606-7618, 1987.

Glassmeier, K.-H.: On the influence of ionospheres with nonuniform conductivity distribution on hydromagnetic waves, J. Geophys., 54, 125-137, 1984.

Glassmeier, K.-H.: Ground-based observations of field-aligned currents in the auroral zone: Methods and results, Ann. Geophys. 3, 115-126, 1987.

Green, D. L., Waters, C. L., Anderson, B. J., Korth, H., and Barnes, R. J.: Comparison of large-scale Birkeland currents determined from Iridium and SuperDARN data, Ann. Geophys., 24, 941- 
959, doi:10.5194/angeo-24-941-2006, 2006.

Green, D. L., Waters, C. L., Korth, H., Anderson, B. J., Ridley, A. J., and Barnes, R. J.: Technique: Large-scale ionospheric conductance estimated from combined satellite and groundbased electromagnetic data, J. Geophys. Res., 112, A05303, doi:10.1029/2006JA012069, 2007.

Greenwald, R. A., Weiss, W., Nielsen, E., and Thomson, N. R.: STARE: A new radar backscatter experiment in northern Scandinavia, Radio Sci., 13, 1021-1039, 1978.

Greenwald, R. A., Baker, K. B., Dudeney, J. R., Pinnock, M., Jones, T. B., Thomas, E. C., Villain, J.-P., Cerisier, J.-C., Senior, C., Hanuise, C., Hundsucker, R. D., Sofko, G., Koehler, J., Nielsen, E., Pellinen, R., Walker, A. D. M., Sato, N., and Yamagishi, H.: DARN $\backslash$ SuperDARN - A global view of the dynamics of highlatitude convection, Space Sci. Rev., 71, 761-796, 1995.

Haines, G. V.: Spherical cap harmonic analysis, J. Geophys. Res., 90, 2583-2591, 1985.

Haines, G. V. and Torta, J. M.: Determination of equivalent current sources from spherical cap harmonic models of geomagnetic field variations, Geophys. J. Int., 118, 499-514, 1994.

Inhester, B., Untiedt, J., Segatz, M., and Kürschner, M.: Direct determination of the local ionospheric Hall conductance distribution from two-dimensional electric and magnetic field data, J. Geophys. Res., 97, 4073-4083, 1992.

Janhunen, P.: On the possibility of using an electromagnetic ionosphere in global MHD simulations, Ann. Geophys., 16, 397-402, doi:10.1007/s00585-998-0397-y, 1998.

Janhunen, P.: Reconstruction of electron precipitation characteristics from a set of multi-wavelength digital all-sky auroral images, J. Geophys. Res., 106, 18505-18516, 2001.

Juusola, L., Amm, O., and Viljanen, A.: One-dimensional spherical elementary current systems and their use for determining ionospheric currents from satellite measurement, Earth Planets Space, 58, 667-678, 2006.

Juusola, L., Amm, O., Kauristie, K., and Viljanen, A.: A model for estimating the relation between the Hall to Pedersen conductance ratio and ground magnetic data derived from CHAMP satellite statistics, Ann. Geophys., 25, 721-736, doi:10.5194/angeo-25721-2007, 2007.

Juusola, L., Kauristie, K., Amm, O., and Ritter, P.: Statistical dependence of auroral ionospheric currents on solar wind and geomagnetic parameters from 5 years of CHAMP satellite data, Ann. Geophys., 27, 1005-1017, doi:10.5194/angeo-27-10052009, 2009.

Kamide, Y., Richmond, A., and Matsushita, S.: Estimation of ionospheric electric fields, ionospheric currents, and field-aligned currents from ground magnetic records, J. Geophys. Res., 86, 801-813, 1981.

Kamide, Y., Sun, W., and Akasofu, S.-I.: The average ionospheric electrodynamics for the different substorm phases, J. Geophys. Res., 101, 99-109, 1996.

Kamide, Y., Kihn, E. A., Ridley, A. J., Cliver, E. W., and Kadowaki, Y.: Real-time specifications of the geospace environment, Space Sci. Rev., 107, 307-316, 2003.

Knipp, D. J., Emery, B. A., Richmond, A. D., Crooker, N. U., Hairston, M. R., Cumnock, J. A., Denig, W. F., Rich, F. J., de la Beaujardiere, O., Ruohoniemi, J. M., Rodger, A. S., Crowley, G., Anh, B.-H., Evans, D. S., Fuller-Rowell, T. J., Friis-Christensen, E., Lockwood, M., Kroehl, H. W., Maclennan, C. G., McEwin,
A., Pellinen, R. J., Morris, R. J., Burns, G., Papitashvili, V., Zaitsev, A., Troshichev, O., Sato, N., Sutcliffe, P., and Tomlinson, L.: Ionospheric convection response to slow, strong variations in a northward Interplanetary Magnetic Field: A case study for January 14, 1988, J. Geophys. Res., 98, 19273-19292, 1993.

Lester, M., Davies, J. A., and Virdi, T. S.: High-latitude Hall and Pedersen conductances during substorm activity in the SUNDIAL-ATLAS campaign, J. Geophys. Res., 101, 2671926728, 1996.

Lummerzheim, D., Rees, M. H., Craven, J. D., and Frank, L. A.: Ionospheric conductances derived from DE-1 auroral images, J. Atmos. Terr. Phys., 53, 281-289, 1991.

Lysak, R.: Magnetosphere-ionosphere coupling by Alfvén waves at midlatitudes, J. Geophys. Res., 109, A07201, doi:10.1029/2004JA010454, 2004.

Lysak, R. and Song, Y.: A three-dimensional model of the propagation of Alfvén waves through the auroral ionosphere: First results, Adv. Space Res., 28, 813-822, 2001.

Matsuo, T., Richmond, A. D., and Lu, G.: Optimal interpolation analysis of high-latitude ionospheric electrodynamics using empirical orthogonal functions: Estimation of dominant modes of variability and temporal scales of large-scale electric fields, J. Geophys. Res., 110, A06301, doi:10.1029/2004JA010531, 2005.

Mersmann, U., Baumjohann, W., Küppers, F., and Lange, K.: Analysis of an eastward electrojet by means of upward continuation of ground-based magnetometer data, J. Geophys., 45, 281-298, 1979.

Milan, S. E., Yeoman, T. K., Lester, M., Thomas, E. C., and Jones, T. B.: Initial backscatter occurrence statistics from the CUTLASS HF radars, Ann. Geophys., 15, 703-718, doi:10.1007/s00585-997-0703-0, 1997.

Murison, M., Richmond, A., Matsushita, S., and Baumjohann, W.: Estimation of ionospheric electric fields and currents from a regional magnetometer array, J. Geophys. Res., 90, 3525-3530, 1985.

Nicolls, M. J. and Heinselman, C. J.: Three-dimensional measurements of traveling ionospheric disturbances with the Poker Flat Incoherent Scatter Radar, Geophys. Res. Lett., 34, L21104, doi:10.1029/2007GL031506, 2007.

Olsen, N.: A new tool for determining ionospheric currents from magnetic satellite data, Geophys. Res. Lett., 23, 3635-3638, 1996.

Parkinson, W. D. and Jones, F. W. : The Geomagnetic Coast Effect, Rev. Geophys. Space Phys., 17, 1999-2015, 1979.

Pulkkinen, A., Amm, O., Viljanen, A., and BEAR Working Group: Ionospheric equivalent current distributions determined with the method of spherical elementary current systems, J. Geophys. Res., 108(A2), 1053, doi:10.1029/2001JA005085, 2003 a.

Pulkkinen, A., Amm, O., Viljanen, A., and BEAR Working Group: Separation of the geomagnetic variation field on the ground into external and internal parts using the spherical elementary current system method, Earth Planets Space, 55, 117-129, 2003b.

Richmond, A. D. and Baumjohann, W.: Three-dimensional analysis of magnetometer array data, J. Geophys., 54, 138-156, 1983.

Richmond, A. D. and Kamide, Y.: Mapping electrodynamic features of the high-latitude ionosphere from localized observations: Technique, J. Geophys. Res., 93, 5741-5759, 1988.

Richmond, A. D., Lu, G., Emery, B. A., and Knipp, D. J.: The AMIE procedure: Prospects for space weather specification and 
prediction, Adv. Space Res., 22, 103-112, 1998.

Ritter, P. and Lühr, H.: Curl-B technique applied to Swarm constellation for determining field-aligned currents, Earth Planets Space, 58, 463-476, 2006.

Ritter, P., Lühr, H., Viljanen, A., Amm, O., Pulkkinen, A., and Sillanpää, I.: Ionospheric currents estimated simultaneously from CHAMP satelliteand IMAGE ground-based magnetic field measurements: a statisticalstudy at auroral latitudes, Ann. Geophys., 22, 417-430, doi:10.5194/angeo-22-417-2004, 2004.

Robinson, R. M., Vondrak, R. R., Miller, K., Dabbs, T., and Hardy, D.: On calculating ionospheric conductances from the flux and energy of precipitating particles, J. Geophys. Res., 92, 25652569, 1987.

Ruohoniemi, J. and Baker, K.: Large-scale imagining of highlatitude convection with Super Dual Auroral Radar Network HF radar observations, J. Geophys. Res., 103, 20797-20811, 1998.

Sciffer, M. D., Waters, C. L., and Menk, F. W.: Propagation of ULF waves through the ionosphere: Inductive effect for oblique magnetic fields, Ann. Geophys., 22, 1155-1169, doi:10.5194/angeo22-1155-2004, 2004.

Senior, A., Kosch, M. J., and Honary, F.: Comparison of methods to determine auroral ionospheric conductances using groundbased optical and riometer data, Ann. Geophys., 26, 3831-3840, doi:10.5194/angeo-26-3831-2008, 2008.

Syrjäsuo, M. T., Pulkkinen, T. I., Janhunen, P., Viljanen, A., Pellinen, R. J., Kauristie, K., Opgenoorth, H. J., Wallman, S., Eglitis, P., Karlsson, P., Amm, O., Nielsen, E., and Thomas, C.: Observations of substorm electrodynamics using the MIRACLE network, in: Substorms-4, edited by: Kokubun, S. and Kamide, Y., Terra Scientific Publishing Company, Tokyo, 111-114, 1998.

Takeda, M.: Effects of the induction electric field on ionospheric current systems driven by field-aligned currents of magnetospheric origin, J. Geophys. Res., 113, A01306, doi:10.1029/2007JA012662, 2008.

Tanskanen, E., Viljanen, A., Pulkkinen, T., Pirjola, R., Häkkinen, L., Pulkkinen, A., and Amm, O.: At substorm onset $40 \%$ of AL comes from underground, J. Geophys. Res., 106(A7), 1311913134, 2001.

Thébault, E., Schott, J. J., and Mandea, M.: Revised spherical cap harmonic analysis (R-SCHA): Validation and properties, J. Geophys. Res., 111, B01102, doi:10.1029/2005JB003836, 2006.

Untiedt, J.: Auroral zone electrodynamics according to combined Scandinavian magnetometer array and STARE backscatter observations, IUGG General Assembly, Hamburg, 1983.
Untiedt, J. and Baumjohann, W.: Studies of polar current systems using the IMS Scandinavian magnetometer array, Space Sci. Rev., 63, 245-390, 1993.

Vanhamäki, H.: Theoretical modeling of ionospheric electrodynamics including induction effects, Finnish Meteorological Institute Contributions, 66, electronic version available at http: //ethesis.helsinki.fi/, 2007.

Vanhamäki, H.: Inductive ionospheric solver for magnetospheric MHD simulations, Ann. Geophys., 29, 97-108, doi:10.5194/angeo-29-97-2011, 2011.

Vanhamäki, H. and Amm, O.: A new method to estimate ionospheric electric fields and currents using data from a local ground magnetometer network, Ann. Geophys., 25, 1141-1156, doi:10.5194/angeo-25-1141-2007, 2007.

Vanhamäki, H., Amm, O., and Viljanen, A.: One-dimensional upward continuation of the ground magnetic field disturbance using elementary current systems, Earth Planets Space, 55, 613-625, 2003.

Vanhamäki, H., Amm, O., and Viljanen, A.: New method for solving inductive electric fields in the non-uniformly conducting ionosphere, Ann. Geophys., 24, 2573-2582, doi:10.5194/angeo24-2573-2006, 2006.

Vanhamäki, H., Amm, O., and Viljanen, A.: Role of inductive electric fields and currents in dynamical ionospheric situations, Ann Geophys., 25, 437-455, doi:10.5194/angeo-25-437-2007, 2007.

Yoshikawa, A. and Itonaga, M.: Reflection of shear Alfvén waves at the ionosphere and the divergent Hall current, Geophys. Res. Lett., 23, 101-104, 1996.

Yoshikawa, A. and Itonaga, M.: The nature of reflection and mode conversion of MHD-waves in the inductive ionosphere: Multistep mode conversion between divergent and rotational electric fields, J. Geophys. Res., 105, 10565-10584, 2000.

Yoshikawa, A., Nakata, H., Nakamizo, A., Uozumi, T., Itonaga, M., Fujita, S., Yumoto, K., and Tanaka, T.: Alfveniccoupling algorithm for global and dynamical magnetosphereionosphere coupled system, J. Geophys. Res., 115, A04211, doi:10.1029/2009JA014924, 2010.

Yumoto, K.: MAGDAS project for Space Weather research and application, Future perspectives of space plasma and particle instrumentation and international collaborations, Proceedings of the International Conference, AIP Conference Proceedings, 1144, 173-178, 2009. 